\title{
A comprehensive portrait of cilia and ciliopathies from a GRISPR-based screen for Hedgehog signaling
}

David K. Breslow ${ }^{1,2,6, *}$, Sascha Hoogendoorn ${ }^{3,6}$, Adam R. Kopp², David W. Morgens ${ }^{4}$, Brandon K. Vu², Kyuho Han ${ }^{4}$, Amy Li $^{4}$, Gaelen T. Hess ${ }^{4}$, Michael C. Bassik ${ }^{4}$, James K. Chen,5,*, Maxence V. Nachury ${ }^{2, *}$

1. Department of Molecular, Cellular and Developmental Biology, Yale University, New Haven, CT 06511

2. Department of Molecular and Cellular Physiology, Stanford University School of Medicine, Stanford, CA 94305

3. Department of Chemical and Systems Biology, Stanford University School of Medicine, Stanford, CA 94305

4. Department of Genetics, Stanford University School of Medicine, Stanford, CA 94305

5. Department of Developmental Biology, Stanford University School of Medicine, Stanford, CA 94305

6. Co-first author

* Correspondence

David K. Breslow

david.breslow@yale.edu

(203) 432-8280

James K. Chen

jameschen@stanford.edu

(650) 725-3582

Maxence V. Nachury

nachury@gmail.com

(650) 721-1999 


\section{Abstract}

2 The primary cilium organizes Hedgehog signaling, shapes embryonic development and is the

3 unifying cause of the ciliopathies. We conducted a functional genomic screen for Hedgehog

4 signaling by engineering antibiotic-based selection of Hedgehog-responsive cells and applying

5 genome-wide CRISPR-mediated gene disruption. The screen robustly identifies factors required

6 for ciliary signaling with few false positives or false negatives. Characterization of hit genes uncovers

7 novel components of several ciliary structures including a protein complex containing $\varepsilon$ - and $\delta$ -

8 tubulin that is required for centriole maintenance. The screen also provides an unbiased tool for

9 classifying ciliopathies and reveals that many forms of congenital heart defects are ciliopathies.

10 Collectively, this screen enables a systematic analysis of ciliary function and of ciliopathies and also

11 defines a versatile platform for dissecting signaling pathways through CRISPR-based screening.

12

13

\section{Keywords}

15 CRISPR, functional genomics, primary cilium, signaling, Hedgehog, centriole, ciliopathy, genetic 16 screen 


\section{Introduction}

The primary cilium is a surface-exposed microtubule-based compartment that serves as an

3 organizing center for diverse signaling pathways ${ }^{1-3}$. Consistent with a central role for cilia in

4 signaling, mutations affecting cilia cause ciliopathies, a group of developmental disorders that

5 includes Joubert Syndrome, Meckel Syndrome (MKS), Nephronophthisis (NPHP), and Bardet-

6 Biedl Syndrome (BBS). The defining symptoms of ciliopathies include skeletal malformations (e.g.

7 polydactyly), mental retardation, sensory defects (e.g. retinal degeneration), obesity, and kidney

8 cysts and are thought to arise from misregulation of ciliary signaling pathways. Over the past fifteen

9 years, advances in human genetics have led to the identification of over 70 ciliopathy disease

10 genes ${ }^{4}$. However, the molecular basis for many ciliopathy cases remains undiagnosed, suggesting

11 that additional ciliopathy genes have yet to be identified ${ }^{5}$. More broadly, many mechanistic aspects

12 of cilium assembly and function remain poorly understood, indicating a need for systematic

13 discovery and characterization of genes that enable ciliary signaling.

14 A key paradigm for ciliary signaling is the vertebrate Hedgehog $(\mathrm{Hh})$ pathway, which

15 exhibits a strict dependence on primary cilia for signaling output ${ }^{3}$. Remarkably, all core

16 components of the Hh signaling machinery - from the receptor PTCH1 to the GLI protein

17 transcriptional effectors - dynamically localize to cilia during signal transduction. Nonetheless, the

18 precise role of cilia in Hh signaling remains elusive, and each successive step in signal transduction

19 is still incompletely understood ${ }^{6}$. Given the key roles of Hh signaling in embryonic development

20 and in cancers such as medulloblastoma and basal cell carcinoma ${ }^{7,8}$, there is a pressing need to fill

21 the gaps in our understanding of Hh signal transduction.

22 Efforts to systematically identify genes needed for cilium assembly or Hh signaling have

23 been reported by a number of groups. However, these studies have relied on arrayed siRNA

24 libraries and exhibit the high rates of false positives and false negatives that are characteristic of

25 RNA interference (RNAi)-based screens ${ }^{9-12}$. Recently, genome-wide screening using the

26 CRISPR/Cas9 system for gene disruption has emerged as a powerful tool for functional

27 genomics ${ }^{13-16}$. CRISPR-based screens have already aided greatly in defining genes required for

28 proliferation of mammalian cells, revealing a core set of essential genes and cancer cell line-specific

29 vulnerabilities. However, the pooled screening format used in these studies requires a means to

30 select for/against or otherwise isolate cells exhibiting the desired phenotype, a technical

31 requirement that has significantly limited the scope of biological applications amenable to pooled 
1 CRISPR-based screens. Indeed, most studies to date have searched for genes that either

2 intrinsically affect cell growth or that affect sensitivity to applied toxins, drugs, or microorganisms ${ }^{17-}$

324

4 Here, we engineered a $\mathrm{Hh}$ pathway-sensitive reporter to enable the systematic 5 identification of genes that participate in Hh signal transduction and primary cilium function.

6 Combining this reporter with a single guide RNA (sgRNA) library targeting the mouse genome,

7 we conducted a CRISPR-based screen that systematically identified ciliary components, Hh

8 signaling machinery, and ciliopathy genes with few false positives or false negatives. Our screen

9 also revealed many genes that had not previously been characterized or linked to ciliary signaling,

10 and we show here that these hits include new components of cilia and centrioles and novel

11 ciliopathy genes. 


\section{Results}

2 Development of a Hh pathway transcriptional reporter for pooled screening

4 a desired phenotype from within a large population of cells. Because ciliary signaling is not

5 intrinsically linked to such a selectable/isolatable phenotype, we engineered a reporter construct

6 that converts Hh signaling into antibiotic resistance (Fig. 1A-B). This transcriptional reporter was

7 introduced into mouse NIH-3T3 fibroblasts, a widely used model cell line for Hh signaling and

8 cilium biology ${ }^{25}$. These cells were then further modified to express the Cas9 endonuclease fused to

$9 \operatorname{BFP}(3 \mathrm{~T} 3-[$ Shh-BlastR;Cas9] cells).

To confirm that our reporter cell line faithfully recapitulates ciliary Hh signaling and to test

11 its suitability for CRISPR-based mutagenesis, we introduced sgRNAs targeting regulators of the

12 canonical Hh pathway (Supplementary Table 1). Smo, a key Hh pathway transducer, and Ift88, an

13 intraflagellar transport (IFT) complex subunit needed for cilium assembly, are required for Hh

14 signaling while GLI3 repressor dampens the Hh response and SUFU prevents Hh signaling in the

15 absence of ligand (Fig. 1c, left). As expected, sgRNAs targeting Smo or Ifto8 severely reduced Sonic

16 Hedgehog N-terminal domain (ShhN)-induced blasticidin resistance, deleting Gli3 potentiated

17 blasticidin resistance in response to ShhN, and targeting Sufu gave rise to ligand-independent

18 blasticidin resistance (Fig. 1c, right). The effects of these sgRNAs on blasticidin resistance were

19 paralleled by concordant changes in endogenous pathway outputs, including the induction of GLI1

20 and the shift from production of GLI3 repressor to GLI3 activator that normally occur upon

21 pathway activation (Supplementary Fig. 1a).

22 Having established a cell line that converts differences in ciliary Hh signaling into different

23 levels of blasticidin resistance, we next tested its suitability for pooled screening. The analysis of

24 our pooled screen is based on quantifying sgRNAs in blasticidin-selected and unselected cell pools

25 to identify sgRNAs that confer a selective advantage or disadvantage (Fig. 1d). We therefore

26 mimicked screening conditions by mixing GFP-marked cells transduced with a Smo-targeting

27 sgRNA with mCherry-marked cells transduced with a portion of our genome-wide sgRNA library.

28 Monitoring $\mathrm{GFP}^{+} /$Smo sgRNA cells by flow cytometry, we found the fraction of Smo sgRNA cells

29 decreased by $>12$-fold and by $>50$-fold after one and two rounds of signaling and selection,

30 respectively (Fig. 1e-f); such changes are readily detectable by deep sequencing. 
Genome-wide screening

We conducted our genome-wide screen using a newly developed sgRNA library targeting

3 the mouse genome ${ }^{26}$. Key features of this library are the use of 10 sgRNAs per gene and the

4 inclusion of $>10,000$ negative control sgRNAs that either do not have targets in the mouse genome

5 or that target "safe" sites with no predicted functional role. We introduced this library into 3T3-

6 [Shh-BlastR;Cas9] cells via lentiviral transduction at low multiplicity of infection and maintained

7 sufficient cell numbers to ensure $\sim 1000 \mathrm{X}$ coverage of the library (due to the large number of cells

8 required, we conducted the screen in four batches using subsets of the library; Supplementary Fig.

9 2a). After sgRNA transduction, cells were exposed to ShhN for $24 \mathrm{~h}$ to fully stimulate Hh signaling,

10 split into separate blastidicin-selected and unselected pools and then subjected to a second cycle of

11 signaling and selection before harvesting and sgRNA counting by deep sequencing (Fig. 1c). Genes

12 affecting ciliary signaling were identified by comparing sequencing reads in the blastidicin-selected

13 versus unselected cell pools at the end of the experiment, while genes affecting proliferation were

14 identified by comparing the plasmid sgRNA library used for lentivirus production to the sgRNA

15 library after 15 days growth in the absence of blasticidin. For statistical analysis, a maximum

16 likelihood method termed casTLE ${ }^{27}$ was used to determine a $P$ value for each gene from the

17 changes in abundance of the corresponding sgRNAs. In addition, the casTLE method also

18 estimates an effect size corresponding to the apparent strength of the phenotype caused by

19 knockout of a given gene.

Assessment of screen performance

To evaluate the quality of our screen, we first assessed its performance in detecting genes

23 affecting growth. This readout is independent of our reporter-based selection strategy and enables

24 comparisons to other growth-based screens. It also provides an opportunity to evaluate the

25 performance of our sgRNA library, as this study represents its first use in a genome-wide screen.

26 Using reference positive and negative essential gene $\operatorname{sets}^{28}$, we found that our screen detected

27 growth-affecting genes with few false positives or false negatives, identifying $>90 \%$ of essential

28 genes with a 5\% false discovery rate (FDR) (Supplementary Fig. 2b and Supplementary Tables 2-

29 3). This performance is comparable to that seen recently with two other libraries ${ }^{19,21}$.

We next evaluated the ability of the blasticidin-based screen to identify genes known to

31 participate in ciliary Hh signaling. Initial inspection of screen results for Smo, Ift88, Gli1, Gli3, and 
1 Sufu revealed several sgRNAs targeting each gene that were depleted or enriched as expected upon

2 blasticidin selection (Fig. 2a). Virtually all known Hh signaling components were among the top

3 hits, including positive regulators Smo, Grk2, Kif7, Prkar1a, Gli1, and Gli2 and negative regulators

4 Ptch1, Adcy6, Gsk3b, Sufu, and Gli3 (Fig. 2b and Supplementary Table 4).

$5 \quad$ In addition to identifying Hh pathway genes, our screen recovered many hits required for

6 the assembly and function of primary cilia. Remarkably, these hits encompass nearly all functional

7 and structural elements of cilia, highlighting the diverse features of cilia needed for effective

8 signaling (Fig. 2c). For example, several hits encode components of the basal body that nucleates

9 the cilium, the transition fibers that anchor the basal body to the cell surface, the transition zone

10 that gates protein entry into the cilium, the ciliary motors that mediate intraciliary transport, and

11 the IFT complexes that traffic ciliary cargos (Fig. 2c and Supplementary Table 4). The detection

12 of nearly all IFT-A and IFT-B genes as hits also underscores the low rate of false negatives.

We observed no apparent correlation between growth and signaling phenotypes, indicating

14 that our antibiotic selection strategy is not biased by general effects on proliferation (Supplementary

15 Fig. 2c). However, because low sgRNA counts were commonly obtained for genes with the

16 strongest growth defects, the estimated signaling phenotypes for these genes may be less reliable

17 and were considered separately. In total, we obtained 472 hits at a 10\% FDR and 969 hits at a

$1820 \% \mathrm{FDR}$, and the majority of these hits led to decreased signaling rather than increased signaling

19 (Fig. 2c). Gene ontology (GO) term analysis using DAVID ${ }^{29}$ revealed that the top 472 hit genes

20 were significantly enriched for many expected functional categories (e.g. cilium morphogenesis, $P$

$21<1 \times 10^{-60}$; Smoothened signaling pathway, $\left.P<1 \times 10^{-31}\right)$ as well as some novel categories, indicating

22 new avenues for investigation (Fig. 2d and Supplementary Table 5). In some cases, corroborating

23 reports support these new connections: for example, mouse mutants for two hit genes that mediate

24 diphthamide modification exhibit Hh pathway-related phenotypes such as polydactyly ${ }^{30,31}$. DPH1

25 mutations have also been shown to cause a poorly characterized syndrome with several ciliopathy-

26 like features including cerebellar vermis hypoplasia, craniofacial malformations, and cardiac

27 defects $^{32}$. Other unexpected gene categories, such as endosome-to-lysosome transport or the Ras

28 signaling pathway, will require further investigation.

29 We next sought to use reference sets of expected hit and non-hit genes to quantitatively

30 assess screen performance. To this end, we curated a set of ciliogenesis reference genes ${ }^{9}$ to generate

31 a list of 130 expected hits (Supplementary Table 3). For expected non-hits, we used 1386 olfactory 
1 and vomeronasal receptor genes, as they are likely not expressed in cultured fibroblasts. Using

2 these reference gene sets, we calculated precision-recall and receiver operating characteristic

3 (ROG) curves (Fig. 3a) from the $P$ values generated by casTLE. Both performance metrics show a

4 high area under the curve (0.802 for precision-recall, 0.892 for ROC), demonstrating that our

5 screen detects hits with high sensitivity and precision (Fig. 3a).

As a second means of evaluation, we compared our ability to detect expected hit genes to that of three related screens. These studies used arrayed siRNA-based screening to study either Hh

8 signaling using a luciferase reporter ${ }^{11}$ or ciliogenesis using microscopy-based measures of ciliary

9 markers ${ }^{9,10}$. While there are notable differences among the screens (e.g. Roosing et al. incorporated data from other sources such as gene expression to score hits ${ }^{9}$ ), they each defined a number of hit

11 genes similar to (or greater than) our screen, thus making it straightforward to compare

12 performance. Overall, we detected the vast majority of expected hits across functional categories

13 ranging from Hh pathway components to ciliopathy genes. Furthermore, even though our screen

14 was focused on Hh signaling, we detected a greater fraction of ciliary hits than the ciliogenesis

15 screens across categories including IFT subunits, ciliary motors, and nearly all classes of ciliopathy

16 genes (Fig. 3b-c, Supplementary Fig. 3a and Supplementary Table 4). One exception however is

17 the group of genes mutated in NPHP, where we found few hit genes (particularly when analyzing

18 genes mutated exclusively in NPHP). While the basis of this finding warrants further study, it raises

19 the possibility that NPHP pathophysiology may be distinct from that of other ciliopathies.

As a final assessment of our screening platform, we evaluated reproducibility across

21 replicate screens. We observed high concordance among hits for the 95 genes that were measured

22 in two different batches of the screen (Supplementary Fig. 3b), with 50 of 54 screen hits also scoring

23 as hits in the second batch. Similarly, strong overlap in hits was found for 263 genes that were

24 screened using two similar but distinct means to initiate Hh signaling: addition of PTCH1 ligand

25 (ShhN) or SMO agonist (SAG) (Supplementary Fig. 3c). This reproducibility makes it possible to

26 directly compare screens and pinpoint genes acting at specific steps in Hh signal transduction. For

27 example, Gas 1 was a hit in the ShhN screen but not in the SAG screen, a result in agreement with

28 GAS1's known function as a Shh co-receptor ${ }^{33,34}$. Overall, our results indicate that combining

29 CRISPR-based screening with a pathway-specific transcriptional reporter is a powerful strategy

30 for functional genomics. 
Identification of new ciliary components

The effectiveness of our screen in identifying genes known to participate in cilium function corroborates the essentiality of this organelle for vertebrate Hh signaling, and our success in

4 identifying the known $\mathrm{Hh}$ signal transduction machinery suggests that novel $\mathrm{Hh}$ pathway 5 components will be found among the many hits. Given that there are likely many more cilium6 related genes than core Hh pathway components, we set out to characterize the roles of six hit 7 genes in cilium biology as a way to further establish the value of our screen.

8 We first focused on Fam92a and Ttc23 because their gene products contain domains 9 associated with membrane trafficking. For Fam92a, we confirmed our pooled screen results in cells transduced with individually cloned sgRNAs, finding that Fam92a knockout caused a strong defect

11 in inducible blasticidin resistance (Supplementary Fig. 4a). This defect was also seen for induction 12 of luciferase from a GLI binding site reporter and could be rescued by re-introduction of sgRNA13 resistant Fam92a (Fig. 4a), indicating that the phenotype is both specific and independent of the

14 blasticidin-based readout. Notably, ciliogenesis was also severely reduced in Fam92a knockout cell 15 pools (Fig. 4b). To gain further insight into Fam92a function, we identified FAM92A-associated 16 proteins using a cell line expressing FAM92A-LAP (localization and affinity purification tag 17 consisting of S-tag-HRV3C-GFP). Affinity purification of FAM92-LAP specifically recovered the 18 known transition zone components CBY1 and DZIP1L (Fig. 4c and Supplementary Table 6) ${ }^{35-37 .}$ 19 Consistent with this finding, we observed prominent FAM92A localization at the transition zone 20 using both an antibody whose specificity we validated in knockout cells (Supplementary Fig. 4b-c) 21 and the FAM92A-LAP cell line (Fig. 4d). While this work was in progress, another group 22 independently identified FAM92A as a transition zone protein contributing to ciliogenesis, 23 corroborating our studies ${ }^{38}$.

24 To characterize the TPR domain-containing protein TTC23, we analyzed TTC2325 interacting proteins by affinity purification and mass spectrometry. Notably, the most prominent 26 TTC23-associated proteins were IQGE and EFCAB7, which represent the non-transmembrane 27 components of the Ellis-van Creveld $(\mathrm{EvC})$ zone. The EvC zone is a proximal region of the cilium 28 that is important for $\mathrm{Hh}$ signaling but dispensable for cilium assembly 39,40 (Fig. 4e and 29 Supplementary Table 6). Of the four proteins -EVG, EVG2, IQCE, and EFGAB7- that are 30 known to localize to the $\mathrm{EvC}$ zone, $E V C$ and $E V C 2$ are also mutated in the ciliopathy Ellis-van 31 Creveld syndrome ${ }^{41}$. Consistent with the biochemical interactions we identified, TTC23-LAP co- 
1 localized with EVG and IQCE at the EvC zone (Fig. 4f and Supplementary Fig. 4d). To assess

2 TTC23 function, we examined Ttc23 knockout cells, which showed a moderate reduction in

3 inducible blasticidin resistance but no defect in cilium assembly (Supplementary Fig. 4a,e). Ttc23

4 knockout cells also showed reduced localization of IQCE and EVC to the EvG zone (Fig. 4g and

5 Supplementary Fig. 4f-g); conversely, Iqce RNAi led to a strong decrease in TTC23-LAP

6 localization to the EvC zone (Fig. 4h). Together these results establish TTC23 as a novel EvG zone

7 component that participates in Hh signaling.

$9 \quad$ Identification of novel disease genes

10 Because the vast majority of ciliopathy genes were hits in the screen, we asked whether 11 uncharacterized hit genes may be mutated in ciliopathies of previously unknown etiology. We first

12 examined Txndc15, a screen hit that encodes a thioredoxin domain-containing transmembrane 13 protein of unknown function. Notably, a previous analysis of MKS patients identified a family with

14 a TXNDC15 mutation ${ }^{42}$. However, other candidate genes could not be ruled out, and an EXOC4 15 variant was favored as the causative mutation. To investigate whether loss of TXNDC15 might 16 cause a ciliopathy, we analyzed Txndc15 knockout cells using the luciferase-based reporter assay, 17 finding a clear defect in Hh signaling. Furthermore, introduction of wildtype Txndc15 rescued this 18 defect, whereas cDNA encoding the mutant allele found in MKS patients behaved like a null allele 19 (Fig. 5a). We also found that cilia in Txndc15 knockout cells exhibited increased variability in length 20 and decreased levels of the ciliary GTPase ARL13B (Fig. 5b and Supplementary Fig. 5a-b). Thus,

21 TXNDC15 likely represents a novel MKS gene, and our screening system can provide a 22 straightforward means to functionally test candidate mutations identified in patients. Consistent 23 with our findings, a very recent follow-up study has identified additional MKS families with 24 TXNDC15 mutations ${ }^{43}$.

25 Similarly, the finding that Armc9 is a hit in our screen raises the possibility that it is a 26 causative ciliopathy gene. Recently, Kar et al. reported that individuals with a homozygous splice 27 site mutation in ARMC9 present with mental retardation, polydactyly, and ptosis ${ }^{44}$ but rejected a 28 diagnosis of Bardet-Biedl syndrome ${ }^{45}$. We found that cilia from $\mathrm{Armc}^{-/-}$cells were short and 29 exhibited reduced levels of acetylated and polyglutamylated tubulin (Fig. 5c and Supplementary 30 Fig. 5c). Furthermore, ARMC9-3xFLAG prominently localized to the proximal region of cilia 31 when stably expressed in IMCD3 cells. Notably, stimulation of Hh signaling led to relocalization 
1 of ARMC9 towards the ciliary tip within $6 \mathrm{hr}$ before a gradual return to its original proximally

2 biased localization, suggesting that ARMC9 may become ectocytosed at later time points ${ }^{46}$ (Fig.

3 5d-e). Furthermore, signaling-induced tip accumulation of GLI2 and GLI3 was reduced in Armc9

4 mutant cells (Fig. 5f and Supplementary Fig. 5d) but SMO translocation to cilia was intact

5 (Supplementary Fig. 5e), suggesting that ARMC9 specifically participates in the trafficking and/or

6 retention of GLI proteins at the ciliary tip. Collectively, these findings demonstrate that ARMC9

7 is a ciliary signaling factor and suggest that ARMC9 is a novel ciliopathy gene.

8 The observation that ARMC9- (and DPH1-) based syndromes likely represent unrecognized

9 ciliopathies led us to ask whether our screen could help classify other genetic disorders as

10 ciliopathies. Consistent with this possibility, the peptidyl-prolyl isomerase Cwc27 was a hit in our

11 screen and was recently identified as a retinitis pigmentosa $(\mathrm{RP})$ gene $^{47}$. Although this syndrome

12 was not recognized as a ciliopathy, the spectrum of $C W C 27$ pathologies reported includes

13 canonical ciliopathy symptoms (craniofacial abnormalities, short stature, brachydactyly, and

14 developmental delay), and we therefore suggest that $C W C 27$-associated disease is a ciliopathy.

15 Similarly, mutations in genes encoding the INTS1 and INTS8 subunits of the Integrator complex

16 were recently described in individuals with a neurodevelopmental disorder with additional facial

17 and skeletal malformations commonly seen in ciliopathies ${ }^{48}$. As the Integrator genes Ints6 and

18 Ints 10 are hits in our screen, disorders due to defects in Integrator complex function may also stem

19 from altered ciliary signaling.

These cases of individual disorders that can now be classified as likely ciliopathies led us to

21 ask whether systematic efforts to map disease genes might reveal broader commonalities with our

22 screen hits. Strikingly, we found that screen hits ANKRD11, CDK13, CHD4, FOXP1, KMT2D, and

23 PRKD1 were all recently identified in a large-scale exome sequencing study of patients with

24 congenital heart defects $(\mathrm{CHD})^{49}$. The significant overlap in these two unbiased datasets $(P=6.11$

$25 \times 10^{-4}$ ) provides compelling evidence that defective ciliary signaling may be a prevalent cause of

26 CHDs. Moreover, mutations in these genes appear to cause bona fide ciliopathies, as patients also

27 exhibit ciliopathy symptoms including craniofacial abnormalities and developmental delay

28 (ANKRD11, CDK13, CHD4, FOXP1, KMT2D, PRKD1), dysgenesis of the corpus callosum (CDK13),

29 polydactyly (CHD4), obesity (ANKRD11), and craniofacial malformations (ANKRD11 and

$30 K M T 2 D)^{4,49,50}$ (Fig. 5g). A link between primary cilia and heart defects was reported in a recent 
1 forward genetic study in mice ${ }^{51}$, and our screen now extends this finding to include several human

2 disease cases.

A new protein complex for centriole stability

Unexpectedly, our screen hits encode not only canonical ciliary proteins but also centriolar

6 proteins not previously linked to ciliary Hh signaling. Given the roles of hits such as CEP19,

7 CEP44, GEP120, and CEP295 at centrioles, we considered whether other hits might also have

8 centriolar functions. Indeed, the uncharacterized hit 1600002H07Rik (human C16orf59) localized

9 to centrioles when stably expressed in IMCD3 cells (Fig. 6a). We performed affinity purifications

10 and found that 1600002H07Rik-LAP co-purified with the uncharacterized protein

11 4930427A07Rik (human C14orf80) and the distant $\alpha / \beta$-tubulin relatives $\varepsilon$-tubulin (TUBE1) and

$12 \delta$-tubulin (TUBD1) (Fig. 6b). All four of these gene products are also among the top-scoring hits in

13 our screen, and $\varepsilon$-tubulin and $\delta$-tubulin have previously been linked to centriole assembly and

14 maintenance ${ }^{52-56}$. We therefore propose to name 4930427A07Rik/C14orfo0 and

$151600002 H 07$ Rik/C16orf59 as Ted1 and Ted2, respectively, for their functional link and biochemical

16 association with tubulins epsilon and delta.

17 Our mass spectrometry analysis of TED2-associated proteins revealed approximately

18 stoichiometric amounts of TED1, TED2, $\varepsilon$-tubulin, and $\delta$-tubulin, as seen by comparison of the

19 normalized spectral abundance factors (Fig. 6b and Supplementary Table 6). We also noted lower

20 amounts of co-purifying $\alpha / \beta$-tubulin and CENPJ/CPAP, a centriolar regulator of microtubule

21 dynamics ${ }^{57-59}$. To corroborate these mass spectrometry analyses, we confirmed co-purification of

22 TUBD1 and TUBE1 by Western blot, readily detecting these proteins in our TED2-LAP

23 purification but not in a negative control purification (Supplementary Fig. 6a). We also tested for

24 an interaction between TED1 and TED2 by co-transfection and co-immunoprecipitation. Not

25 only did we detect a specific interaction between these proteins, we found that TED1 and TED2

26 mutually stabilize their expression levels, leading to notably higher expression when expressed

27 together versus individually (Fig. 6c).

28 To functionally characterize Ted1 and Ted2, we first examined knockout cells generated

29 using two sgRNAs targeting each gene. Strikingly, we found that Ted1 and Ted2 knockout cell pools

30 were almost completely devoid of centrioles, as assessed by staining with antibodies to centrin,

31 ninein, polyglutamylated tubulin or $\gamma$-tubulin (Fig. 7a-b). Congruently, mutant cells also lacked 
1 cilia (Fig. 7a) and had strong defects in Hh pathway reporter induction and GLI protein regulation

2 (Supplementary Fig. 6b-c). We also noted that Ted1 and Ted2 mutants exhibited a mild growth

3 defect (Supplementary Table 3), which is consistent with recent evidence that NIH-3T3 cells

4 lacking centrioles can continue to proliferate, albeit at a reduced rate ${ }^{60}$. By contrast, in other cell

5 types, a p53-dependent arrest prevents proliferation in the absence of centrioles. These

6 observations prompted us to investigate whether loss of Ted1 or Ted2 has varying effects on

7 proliferation across different cell types, and whether such phenotypic variability could enable

8 predictive identification of genes with similar function. We therefore examined a recently published

9 collection of CRISPR-based growth screens conducted in 33 different human cell lines and used

10 hierarchical clustering to group genes based on the similarity of their cell type-specific growth

11 phenotypes ${ }^{61}$. Strikingly, this unbiased approach placed Ted1, Ted2, and Tube1 in a single cluster

12 (Fig. 7b and Supplementary Table 7), suggesting that they have a highly similar function in

13 addition to encoding components of a shared protein complex. We note that TUBD1 did not co-

14 cluster but was likely not effectively targeted by this sgRNA library.

To better understand the basis for centriole loss in Ted1 and Ted2 mutants, we examined centrioles in cells at different stages of the cell cycle. Surprisingly, while mutant cells typically had

17 zero or one centriole in interphase, nearly all mitotic cells had excess centrioles (more than four),

18 suggestive of de novo centriole formation before mitotic entry (Fig. 7c-d and Supplementary Fig. 6d).

19 By contrast, cells exiting mitosis more closely resembled interphase cells, again showing

20 significantly decreased centriole numbers compared to negative control sgRNA-expressing cells

21 (Fig. 7d). Taken together, these observations suggest that Ted1 and Ted2 are dispensable for

22 centriole biogenesis but required for centriole stability, with newly generated centrioles rapidly lost

23 as cells exit mitosis.

24 While the biochemical activities of $\varepsilon$-tubulin and $\delta$-tubulin remain elusive, very recently

25 TUBE1- and TUBD1-deficient human RPE1 cells were found to frequently lack centrioles and

26 undergo cycles of de novo centriole biogenesis followed by disappearance of these centriole-like

27 structures during progression through the cell cycle (J. Wang and T. Stearns, personal

28 communication). This phenotype echoes our findings in Ted1 and Ted2 mutant cells, and together

29 with our biochemical findings indicates that the TED complex is required for centriole stability. 


\section{Discussion}

2 Here we present a new functional screening platform that pairs a pathway-specific

3 selectable reporter with genome-wide CRISPR-based gene disruption. Applying these

4 technologies to systematically investigate cilium-dependent $\mathrm{Hh}$ signaling, we obtain a

5 comprehensive portrait of cilium biology that identifies hit genes with high sensitivity and

6 specificity, surpassing the performance seen in related siRNA-based arrayed screens. As a result,

7 we identify many novel hit genes, and we show that a number of previously uncharacterized gene

8 products have diverse functions in cilium assembly and ciliary signaling.

Screen performance and CRISPR-based screening

Several factors likely contributed to the quality of our screen, including the pooled screening format, the use of CRISPR for gene disruption, and a newly designed sgRNA library.

13 Given the strong influence of cell confluence on cilium assembly and $\mathrm{Hh}$ signaling, the

14 homogeneous growth conditions afforded by pooled screening were likely a major advantage. In

15 an arrayed format, perturbations causing growth defects can indirectly affect ciliogenesis by

16 decreasing cell density, thus generating false positives that need to be filtered out ${ }^{10}$. Pooled screens

17 achieve confluence regardless of genotype, and thus we can successfully identify hits that have

18 moderate proliferation defects, including the four components of the TED complex.

Another key feature of our screen is the use of CRISPR-based gene disruption. The strong phenotypes produced by CRISPR/Cas9 likely made it possible to detect hit genes in cases where

partial knockdown by RNAi or CRISPR interference (CRISPRi) might have failed to produce a

22 detectable phenotype. A potential caveat of our approach is a decreased ability to detect hits among

23 genes that are strictly required for cell viability. However, because ciliary signaling is dispensable

24 for growth of cultured cells, this issue likely had a limited impact on our screen. It is also important

25 to note that the allelic series achievable with RNAi and CRISPRi may be preferable when

26 screening for phenotypic modifiers, as in chemical genetic or genetic interaction analyses.

27 The strong performance of CRISPR-based screening may also be attributable to our use

28 of an sgRNA library comprised of many highly active sgRNAs with few off-target effects. High on-

29 target activity is especially important for detecting hits in dropout-based screens (in which hits

30 become depleted) and was achieved by using $10 \mathrm{sgRNAs}$ per gene and by optimizing the stability

31 of Cas9 expression. A second benefit of using $10 \mathrm{sgRNAs}$ per gene is the increased statistical power 
1 achieved when multiple effective sgRNAs are found targeting a single gene. Indeed, for hit genes

2 such as Dync2h1, Tmem107, Ift80, B9d1, and Grk2, at least 7 out of $10 \mathrm{sgRNAs}$ were depleted more

3 than 4-fold (and up to 45-fold), leading to high statistical confidence. While other genes may not

4 have been targeted as efficiently, the use of 10 elements per gene strongly increases the statistical

5 power of hit gene detection.

A final important feature of our screening platform is that it can readily be extended to

7 other biological processes. To date, most CRISPR-based screens have been limited to situations

8 where the phenotype of interest directly affects cell viability, such as defining factors affecting cell

9 proliferation or resistance to toxins, drugs, or microorganisms. By using a reporter-based selectable marker, our approach allows powerful screening tools to be applied to virtually any process with a

11 well-defined transcriptional response.

New insights into cilium biology, Hh signaling, and ciliopathies

The combined benefits of our pooled CRISPR-based screening approach enabled us to generate a rich dataset that we anticipate will be a lasting resource in the field, both for dissecting the basic mechanisms of ciliary signaling and also for defining new ciliopathy genes and potential

17 therapeutic targets in Hh-driven cancers. While prior siRNA-based screens have contributed to our understanding of cilium function and Hh signaling, many of these datasets suffer from false positives or false negatives that limit their utility. In addition, most screens focused on cilium assembly rather than Hh signaling and thus do not identify Hh pathway components. Roosing et al. ${ }^{9}$ improved their ciliogenesis screen results by using known positive and negative gene sets to

22 help classify hits and by incorporating gene expression datasets in addition to their microscopy-

23 based screen data. Our screening approach achieved high performance without dependence on

24 other data sources, which may not always be available, or on a priori definition of hits, which could

25 bias discovery of new hit classes. Our screens are also highly reproducible, thereby enabling 26 comparative screening approaches that will be instrumental in uncovering novel factors acting at 27 specific steps in Hh signaling, in particular the regulation of SMO by PTCH1, a poorly understood 28 yet critical aspect of Hh signaling.

29 The practical value of our screen is demonstrated by the discovery of new genes that 30 participate in ciliary signaling and new candidate ciliopathy genes. While the precise roles of 31 FAM92A at the transition zone and TTC23 at the EvC zone will require further study, our screen 
1 demonstrates that new components remain to be identified even for these well-studied ciliary

2 structures. Similarly, our analyses of TXNDC15, ARMC9, CWC27, DPH1, INTS6, INTS10,

3 ANKRD11, CDK13, CHD4, FOXP1, PRKD1, and KTM2D illustrate that screen hits can help to

4 identify a ciliopathy-causing gene from a short list of variants, as is frequently the case in modern

5 genome sequencing of small pedigrees, and to classify new genetic syndromes as disorders of ciliary

6 signaling. Indeed, with the exception of $T X N D C 15$, all of the aforementioned genes had been

7 previously linked to disease states (e.g. KMT2D and Kabuki Syndrome, OMIM 147920; ANKRD11

8 and KBG Syndrome, OMIM 148050; DPH1 and Loucks-Innes Syndrome, OMIM 616901)

9 without a potential role for cilia described. Among the syndromes caused by mutations in these

10 genes, it is striking that the most prevalent feature is CHD. Our screen thus provides unbiased

11 evidence that several CHD cases are bona fide ciliopathies, thereby building upon similar

12 connections observed in mice and motivating future investigations by human geneticists and

13 developmental biologists.

14 It is also noteworthy that our screen hits include the vast majority of known ciliopathy genes

15 but not those primarily linked to kidney pathology (with or without laterality defects). In particular,

16 the NPHP genes that are associated with the Inversin compartment (INVS, NPHP3, ANKS6, NEK 8 )

17 and the polycystic kidney disease genes PKD1, PKD2, and PKHD1 are all non-hits. This finding

18 suggests that these renal diseases are mechanistically distinct from other ciliopathies, likely acting

19 independently of Hh signaling and possibly even involving pathomechanisms independent of

20 cilia ${ }^{62}$. By capturing a comprehensive and unbiased picture of cilium-based signaling, our screen

21 refines the classification of ciliopathies. More broadly, as genome sequencing reveals disease-

22 associated variants at ever-growing rates, genome-wide functional studies such as that presented

23 here will become a powerful resource to distinguish disease-causing mutations from innocuous

24 variants ${ }^{63}$ and to gain insight into underlying disease mechanisms.

25 Our screen also identifies hit genes with highly diverse roles in cilium function and $\mathrm{Hh}$

26 signaling, including several genes that participate in centriole biology. For hits Ted1, Ted2, ع-tubulin

27 and $\delta$-tubulin, we found that these four genes act in concert to ensure centriole stability, as

28 evidenced by the association of their gene products in a complex. This complex appears to be a

29 stoichiometric tetramer and demonstrates that $\varepsilon$ - and $\delta$-tubulin directly associate. While the

30 structural basis for this interaction awaits further study, it is tempting to speculate that $\varepsilon$ - and $\delta$ -

31 tubulin may form a heterodimer analogous to $\alpha / \beta$-tubulin. In addition to the physical association 
1 of TED complex components, deficiency for these genes leads to a remarkably similar pattern of

2 growth phenotypes across cell lines. This observation provides further evidence for a shared

3 function and complements recent work demonstrating the precise functional predictions made

4 possible by CRISPR-based growth profiling ${ }^{19}$.

$5 \quad$ Similar to the aforementioned pattern of cell-type-specific growth phenotypes, Turk et al. ${ }^{64}$

6 recently observed a phylogenetic pattern in which the presence or absence of $\varepsilon$-tubulin in a given

7 species predicts whether $\delta$ - or $\zeta$-tubulin is also present. The evolutionary co-occurrence of these

8 centriolar tubulins prompted us to ask whether Ted1 and Ted2 also share a similar phylogenetic

9 distribution. We found Ted1 and Ted2 homologs in annelids and sea urchin and Ted1 homologs in

10 evolutionarily distant species such as Paramecium tetraurelia and Tetrahymena thermophila

11 (Supplementary Fig. 7a). Consistent with a conserved functional relationship among TED complex

12 components, all of these species also have $\varepsilon-, \delta$ - and/or $\zeta$ - tubulins; conversely, we did not detect

13 Ted1 or Ted2 homologs in any species lacking $\varepsilon-, \delta$ - and $\zeta$ - tubulins. The scope of this analysis was

14 limited by the more rapid divergence seen for Ted1 and Ted2 sequences than for $\varepsilon$-, $\delta$ - and $\zeta$ -

15 tubulins (Supplementary Fig. 7b), but this phylogenetic distribution further supports a shared

16 function and echoes what is seen for $\gamma$-tubulin and subunits of the $\gamma$-tubulin ring complex.

17 Notably, studies across diverse species have implicated $\varepsilon$ - and $\delta$-tubulins in stabilizing

18 centriolar microtubules. For example, defects in Paramecium or Tetrahymena $\varepsilon$-tubulin or in

19 Chlamydomonas reinhardtii $\delta$-tubulin all lead to centrioles lacking the $\mathrm{C}$ and/or $\mathrm{B}$ tubules of the

20 centriolar triplet microtubules ${ }^{53,55,56}$. Consistent with these reports, we find that Ted1 and Ted2

21 knockout cells are markedly deficient in centriole maintenance. We speculate that the centriolar

22 structures formed in Ted1/2-deficient cells are structurally unstable and degenerate during mitotic

23 exit at the time when pericentriolar material (PCM) is stripped from the centrosome and duplicated

24 pairs of centrioles become disengaged. Since removal of the PGM has been recently shown to cause

25 centriolar instability in fly spermatocytes ${ }^{65}$, we consider post-mitotic PCM removal may be a key

26 event contributing to centriole loss in Ted mutants. CENPJ may act together with the TED complex

27 to ensure centriole stability, as CENPJ was found in our TED2 purifications and a mutation in

28 Cenpj/Sas-4 was recently shown to lead to loss of triplet microtubules in Drosophila spermatocytes ${ }^{66}$.

29 Lastly, given that several centriolar genes including CENPF cause microcephaly when mutated ${ }^{67,68}$,

30 TED complex components are potential candidate genes for this neurodevelopmental disorder. 
In summary, we have developed a functional screening platform that provides a valuable

2 resource for the investigation of long-standing questions in Hh signaling and in the biology of

3 primary cilia. By applying and extending these tools, it may now be possible to systematically define

4 vulnerabilities in Hh pathway-driven cancers, to search for suppressors of ciliopathies that may

5 inform treatment, and to identify modifiers of $\mathrm{Hh}$ pathway-inhibiting chemotherapeutics.

6 Integrating this functional genomic approach with complementary insights from proteomics and

7 human genetics promises a rich toolkit for understanding ciliary signaling in health and disease.

8 More broadly, the approach described here makes CRISPR-based screening possible for

9 essentially any biological process with a defined transcriptional output. 


\section{Acknowledgments}

2 We acknowledge members of the Chen and Nachury labs for advice and technical support; S. van

3 Dorp for assistance with image quantification; P. Beachy for NIH-3T3 cells, 8xGli-luciferase

4 reporter plasmid, and ShhN-producing HEK293T cells; K.C. Garcia and G. Crabtree for use of

5 plate readers; C. Bustamante for use of an Illumina sequencer; R. Rohatgi for antibodies to Evc

6 and Iqce; J. Wang and T. Stearns for sharing unpublished results and cDNA for Cbyl; and M.

7 Scott for helpful discussions. This project was supported by NIH Pathway to Independence Award

8 K99HD082280 (D.K.B), Damon Runyon Dale F. Frey Award DFS-11-14 (D.K.B.), seed grants

9 from the Stanford Center for Systems Biology (D.K.B., S.H. and G.T.H.) and Stanford ChEM-H

10 (M.C.B.), an NWO Rubicon Postdoctoral Fellowship (S.H.), National Science Foundation

11 Graduate Research Fellowship DGE-114747 (D.W.M.), a Walter V. and Idun Berry Award

12 (K.H.), T32HG000044 (G.T.H.), DP2 HD08406901 (M.C.B.), R01 GM113100 (J.K.C.), and R01

13 GM089933 (M.V.N.). Cell sorting/flow cytometry was done on instruments in the Stanford

14 Shared FACS Facility, including an instrument supported by NIH shared instrument grant

15 S10RR025518-01. Mass spectrometry analyses were conducted in the Vincent Coates Foundation

16 Mass Spectrometry Laboratory, Stanford University Mass Spectrometry and the Stanford Cancer

17 Institute Proteomics/Mass Spectrometry Shared Resource; these centers are supported by Award

18 S10RR027415 from the National Center for Research Resources and NIH P30 CA124435,

19 respectively. We thank Carsten Carstens, Ben Borgo, Peter Sheffield, and Laurakay Bruhn of

20 Agilent Technologies for cilia-focused oligonucleotide sub-libraries. 


\section{Author Contributions}

2 D.K.B., S.H., J.K.C., and M.V.N. conceived the project with advice from M.C.B. D.K.B. and

3 S.H. developed the Hh pathway reporter screening strategy with assistance from B.K.V. D.W.M.,

4 K.H., A. L., G.T.H, and M.C.B. provided functional genomics expertise, the genome-wide sgRNA

5 library, and software for screen data analysis. D.K.B. conducted the genome-wide screen and

6 screen data analysis with assistance from S.H. and A.R.K. D.K.B., S.H., and A.R.K. functionally

7 characterized hit genes of interest, analyzed data, and prepared figures. D.K.B., S.H., and M.V.N.

8 wrote the manuscript with assistance from M.C.B. and J.K.C. D.K.B, S.H., G.T.H., M.C.B,

9 J.K.C. and M.V.N. provided funding for the project.

10

\section{Competing financial interests}

12 The authors declare no competing financial interests. 
METHODS

2

GONTAGT FOR REAGENT AND RESOURGE SHARING

4 Further information and requests for resources and reagents should be directed to and will be

5 fulfilled by David Breslow (david.breslow@yale.edu).

6

7 EXPERIMENTAL MODELS AND SUBJECT DETAILS

8 Cell lines and cell culture

$9 \quad$ NIH-3T3 and HEK293T cells were grown in high glucose DMEM supplemented with

$1010 \%$ fetal bovine serum (FBS), $2 \mathrm{mM}$ glutamine, $1 \mathrm{mM}$ sodium pyruvate, $10 \mathrm{U} / \mathrm{ml}$ penicillin and

$11 \quad 10 \mu \mathrm{g} / \mathrm{ml}$ streptomycin. Light-II NIH-3T3 cells ${ }^{25}$ were grown in the same medium except with

$1210 \%$ bovine calf serum (BCS), and IMCD3 cells were grown in DMEM/F12 medium with the

13 same FBS, glutamine, penicillin and streptomycin additives. Serum starvation was done with the

14 same media but with $0.5 \%$ FBS for NIH-3T3 cells, 0.5\% BCS for Light-II NIH-3T3 cells, and

$150.2 \%$ FBS for IMCD3 cells. Cells were passaged using 0.05\% trypsin-EDTA. IMCD3 FlpIn cells

16 were provided by Peter Jackson. NIH-3T3 cells were obtained from ATCG. HEK239T-EcR-

17 ShhN cells were provided by Philip Beachy. Cells were confirmed to be mycoplasma free by regular

18 testing with the MycoAlert system (Lonza).

\section{METHOD DETAILS}

21 DNA cloning

22 Individual sgRNAs were cloned by ligating annealed oligonucleotides into pMCB306 or

23 pMCB320 digested with BstXI and Bpu1102I (Fermentas FastDigest enzymes, Thermo Fisher).

24 Ligated products were transformed into Mach1-T1 competent cells (Thermo Fisher) and recovered

25 plasmids were verified by sequencing.

Cilia-focused sgRNA libraries were cloned from oligonucleotide pools (Agilent) as

27 described ${ }^{26}$. Briefly, oligonucleotides were amplified using primer sequences common to each sub-

28 library, digested with BstXI and Bpul 102I, and ligated into pMCB320, followed by transformation

29 into Endura competent cells (Lucigen). DNA was isolated from collected bacterial colonies using a 30 Plasmid Plus Giga kit (Qiagen). 
Individual cDNAs encoding genes of interest were amplified from mouse cDNA or

2 commercially available sources (Dharmacon) and cloned into Gateway Entry vectors either via BP

3 clonase-mediated recombination (Thermo Fisher) or using isothermal assembly. Mutations to

4 introduce resistance to sgRNA-mediated cleavage were introduced by isothermal assembly.

5 Plasmids for expression of tagged genes of interest were generated from Entry vectors by LR

6 clonase-mediated recombination (Thermo Fisher) into Destination vectors encoding C-terminal

7 LAP, 3xFLAG, or 6xMyc tags.

8 Plasmid pHR-Pgk-Cas9-BFP was cloned by digestion of pHR-SFFV-Cas9-BFP (M. Bassik)

9 and replacement of the SFFV promoter with the Pgk promoter amplified from pEFB/FRT-pCrys-

10 APGpr161 NG3-NsiI-pPgk-BirA-ER ${ }^{46}$. Plasmid pGL-8xGli-Bsd-T2A-GFP-Hyg was generated in the

11 pGL4.29-[luc2P/CRE/Hygro] vector (Promega) by replacement of the CRE response element

12 with 8xGli binding sites amplified from pGL3-8xGli-Luc (P. Beachy) and of Luc2P with Bsd-T2A-

13 GFP amplified from pEF5B-FRT-DEST-LAP69.

14

Virus production and cell transduction

VSVG-pseudotyped lentiviral particles were produced by co-transfection of HEK293T

17 with a lentiviral vector (e.g. MCB320) and appropriate packaging plasmids (pMD2.G, pRSV-Rev,

18 pMDLg/RRE for pMCB320 and pMCB306 sgRNAs; pCMV- $\Delta$ R-8.91 and pCMV-VSVG for

19 Pgk-Cas9-BFP). Following transfection using polyethyleneimine (linear, MW 25000,

20 Polysciences), virus-containing supernatant was collected $24 \mathrm{~h}$ later, filtered through a $0.45 \mu \mathrm{m}$

21 cellulose acetate filter, and either used immediately or stored at $-80^{\circ} \mathrm{C}$. For sgRNA libraries, a

22 second harvest of viral medium was performed $24 \mathrm{~h}$ after the initial harvest. For Cas9-containing

23 virus, lentiviral particles were concentrated 20-fold using Lenti-X Concentrator (Clontech) prior

24 to use.

Cells were transduced by addition of viral supernatants diluted to an appropriate titer in

26 growth medium containing $4 \mu \mathrm{g} / \mathrm{ml}$ polybrene (Sigma Aldrich). Following $24 \mathrm{~h}$ incubation at

$2737^{\circ} \mathrm{C}$, virus-containing medium was removed; after an additional $24 \mathrm{~h}$, cells were passaged and,

28 where appropriate, selection for transduced cells was commenced by addition of $2.0 \mu \mathrm{g} / \mathrm{ml}$

29 puromycin (Invivogen). Multiplicity of infection was determined by flow cytometry-based analysis

30 of fluorescent marker expression. 
The HEK239T-EcR-ShhN cell line was used to produce ShhN-containing conditioned medium. Cells were grown to $80 \%$ confluence, medium changed to DMEM with 2\% FBS,

4 followed by collection of conditioned medium after $48 \mathrm{~h}$ and filtration through a Steritop $0.22 \mu \mathrm{m}$

5 filter device (EMD Millipore). The titer of ShhN was determined using NIH-3T3 Light-II 8xGli

6 luciferase reporter cells (see Luciferase reporter assays), and a concentration approximately two-fold

7 over the minimum dilution needed for full luciferase induction was used for further experiments.

$9 \quad$ Flow cytometry and fluorescence-activated cell sorting

Flow cytometry analyses were conducted using a FACSScan (Becton Dickinson) outfitted

11 with lasers provided by Cytek Biosciences. FACS was performed using FACSAria II cell sorters

12 (Becton Dickinson). Flow cytometry and FACS data were analyzed using Flowjo (Treestar) to 13 quantify the intensity of fluorescent protein expression and fraction of marker-positive cells.

Generation of stable cell lines

The 3T3-[Shh-BlastR;Cas9] reporter cell line was generated by using Lipofectamine 2000

17 (Thermo Fisher) to transfect NIH-3T3 cells with pGL-8xGli-Bsd-T2A-GFP-Hyg. Following 18 selection for a hygromycin-resistant cell pool, clonal isolates were obtained by limiting dilution 19 cloning and tested for SAG-induced blastidicin resistance. Lentiviral transduction was then used 20 to introduce Pgk-Cas9-BFP, followed by three rounds of sorting the pool of transduced cells for

21 high-level BFP expression. Notably, we found that other promoters either failed to stably express

22 Cas9 after weeks of passaging or produced an excessive level of Cas9 that led to toxicity (data not 23 shown).

Stable cell lines for affinity purification and localization studies were generated using the

25 FlpIn system (Life Technologies) that relies on FRT-mediated recombination into a single FRT 26 site present in IMCD3 FlpIn cells. Plasmids encoding genes of interest in the pEF5B/FRT-DEST-

27 LAP (or 3xFLAG) vector were transfected into IMCD3 FlpIn cells together with pOG44 Flp 28 recombinase (Life Technologies) using X-tremegene 9 (Roche). Recombined cells pools were 29 obtained following selection with blasticidin (Sigma Aldrich) and were used for further analysis. 
2 medium was then replaced with serum starvation medium with or without pathway agonist (ShhN

3 conditioned medium at appropriate dilution or SAG (synthesized as described ${ }^{70,71}$ ) at $250 \mathrm{nM}$ ).

4 After $24 \mathrm{~h}$, cells were passaged at approximately 1:6 dilution to fresh medium with 10\% FBS,

5 allowed to adhere for $5 \mathrm{~h}$, and then subjected to blasticidin (blasticidin $\mathrm{S}$ hydrochloride, Sigma

6 Aldrich) selection at concentrations ranging from 0.5 to $20 \mu \mathrm{g} / \mathrm{ml}$ for $4 \mathrm{~d}$. Relative viability was

7 determined using the CellTiter-Blue fluorescence-based assay (Promega), and fluorescence read on

8 a SpectraMax Paradigm (Molecular Devices) or Infinite M1000 (Tecan) plate reader.

Genome-wide screening

Genome-wide screening was conducted in four batches of $\sim 45,000-70,000 \mathrm{sgRNAs}^{26}$. For

each batch, lentivirus was produced and titered as described above. 3T3-[Shh-BlastR;Cas9] cells

13 were grown in $15 \mathrm{~cm}$ plates and transduced at a multiplicity of infection of $\sim 0.3$ in sufficient

14 numbers such that there was a $\sim 500: 1$ ratio of transduced cells to sgRNA library elements. Cells

15 were selected with puromycin for $5 \mathrm{~d}$, grown for $3 \mathrm{~d}$ without puromycin, and then plated for

16 signaling, maintaining a 1,000:1 ratio of cells to sgRNAs for these and all subsequent steps. After

17 cells reached confluence, signaling was initiated by addition of serum starvation medium

18 containing ShhN. After $24 \mathrm{~h}$, cells were passaged, allowed to adhere, and then subjected to

19 blasticidin selection for $4 \mathrm{~d}$ at $5 \mu \mathrm{g} / \mathrm{ml}$, a concentration we found sufficient to achieve strong

20 enrichment/depletion of hits without causing sgRNA library bottlenecks due to excess cell death.

21 After passaging cells to blasticidin-free medium, a 'T1' sample was harvested (1000-fold more cells

22 than sgRNAs) and remaining cells were passaged once more before seeding for a second round of

23 signaling and selection. The final 'T2' cell sample was collected following $4 \mathrm{~d}$ blasticidin selection

24 and one additional passage in the absence of blasticidin. Unselected control cells were also

25 propagated through the entire experiment and harvested at equivalent timepoints.

Screens using the cilia/Hh pathway-focused library were conducted as above except that

27 a variant blasticidin reporter cell line was used in which Cas9-BFP was expressed using the

28 shortened EF1 $\alpha$ promoter rather than the Pgk promoter. Because some Cas9-negative cells

29 accumulated during the course of the experiment, the final blasticidin-selected and unselected cells

30 were FACS-sorted for BFP-positive cells bearing Cas9. 
To process cell samples for sgRNA sequencing, genomic DNA was first isolated using a

2 QiaAmp DNA Blood Maxi kit or QiaAmp DNA mini kit (Qiagen) depending on the scale of the

3 screen batch. Genomic DNA was then amplified using Herculase II polymerase (Agilent) as

4 described ${ }^{17}$, first using outer primers to amplify the sgRNA cassette from nearly all genomic DNA

5 recovered, then inner primers to amplify a portion of the initial PCR product while introducing

6 sample barcodes and adapters for Illumina sequencing (Supplementary Table 9). Gel purified PGR

7 products were quantified by Qubit 2.0 fluorometer (Thermo Fisher) using the dsDNA HS kit

8 (Thermo Fisher) and pooled for sequencing. Deep sequencing was performed on a NextSeq 500

9 sequencer with high-output v2 kits (Illumina) to obtain an approximately 500-fold excess of reads

10 to sgRNA library elements. Sequencing was performed using custom primers to read the sgRNA

11 protospacer and sample barcode.

Luciferase reporter assays

14 Luciferase reporter assays were conducted using 3T3-[Shh-BlastR;Cas9] cells co-

15 transfected with pGL3-8xGli-Firefly-luciferase and pGL3-SV40-Renilla-luciferase. Cells were

16 transfected using Mirus TransIT-2020 (Mirus Bio) $24 \mathrm{~h}$ after plating with luciferase plasmids and

17 either control GFP-containing plasmid (pEF5B-FRT-GFP-FKBP) ${ }^{72}$ or a plasmid encoding a gene

18 of interest. Nearly confluent cells were switched to serum starvation medium with or without

19 pathway agonist $24 \mathrm{~h}$ later, and allowed to signal for 24-30 h. Alternatively, luciferase assays for

20 titering ShhN-conditioned medium were performed with the NIH-3T3 Light-II cell line, which

21 has stably integrated versions of GLI-driven firefly luciferase and constitutively expressed Renilla

22 luciferase reporters. After signaling, cells were lysed in lysis buffer (12.5 mM Tris pH 7.4, 4\%

23 glycerol, 0.5\% Triton X-100, $0.5 \mathrm{mg} / \mathrm{mL}$ BSA, $1 \mathrm{mM}$ EGTA, $1 \mathrm{mM}$ DTT) and a dual luciferase

24 measurement performed using a Modulus microplate luminometer (Turner Biosystems).

Immunofluorescence and localization studies

For localization studies, IMCD3 FlpIn or 3T3-[Shh-BlastR;Cas9] cells were first plated on acid-washed $13 \mathrm{~mm}$ round \#1.5 coverslips (additionally coated with poly-L-lysine for NIH-3T3

29 cells). After $24 \mathrm{~h}$, cells were transfected with siRNAs (see Supplementary Table 9 for sequences)

30 using Lipofectamine RNAiMAX (Thermo Fisher) or with plasmids using Fugene 6 (Promega).

31 Cells were serum starved for $24 \mathrm{~h}$ if needed and then fixed using 4\% paraformaldehyde, ice-cold 
1 methanol, or both in succession. For GLI2/GLI3/SMO trafficking assays, cells were serum-

2 starved for $20 \mathrm{~h}$, followed by 5-6 h incubation in the presence or absence of the appropriate amount

3 of ShhN-conditioned medium. For analysis of TED2-LAP localization, cells were pre-extracted

4 prior to methanol fixation via a one-minute exposure to PHEM buffer (60 mM PIPES, $25 \mathrm{mM}$

5 HEPES, 4 mM MgSO 4,10 mM EGTA, pH 7.0) with $0.2 \%$ TritonX-100.

$6 \quad$ Fixed coverslips were blocked using PBS with 5\% BSA and 5\% normal donkey serum,

7 permeabilized if needed using PBS with $0.1 \%$ Triton X-100, and then incubated with appropriate

8 primary and secondary antibodies (see Supplementary Table 8 for primary antibodies used).

9 Coverslips were then either stained with Hoechst DNA dye, and mounted on slides using

10 Fluoromount-G mounting medium (Electron Microscopy Sciences), or directly mounted using

11 ProLong Gold antifade reagent with DAPI (Life Technologies).

12 Coverslips were imaged at $60 \mathrm{x}$ or $63 \mathrm{x}$ magnification using one of three microscope systems:

13 an Axio Imager.M1 (Carl Zeiss) equipped with SlideBook v6 software, an LED light source

14 (Intelligent Imaging Innovations) and a Prime 95b sCMOS camera (Photometrics); an Axio

15 Imager.M1 (Carl Zeiss) equipped with SlideBook v5 software, a Lambda XL light source (Sutter

16 instruments) and CoolSNAP HQ ${ }^{2}$ CCD camera (Photometrics), or a DeltaVision Elite imaging

17 system equipped with SoftWoRx software, an LED light source, and sCMOS camera (Applied

18 Precision). Z-stacks were acquired at 250-500 nm intervals and deconvolved as needed using

19 Slidebook 6.0 or SoftWoRx softwares.

Co-transfection and co-immunoprecipitation

HEK293T cells were co-transfected with Ted1, Ted2 or TagRFP plasmids using Fugene 6,

23 collected after $48 \mathrm{~h}$, and lysed on ice in CoIP buffer (50 mM Tris pH 7.4, $150 \mathrm{mM} \mathrm{NaCl}, 1 \%$

24 Triton X-100, 1X DTT, 1X LPB) supplemented with protease inhibitors. Lysates were cleared by

25 centrifugation at 20,000 x $g$ for $20 \mathrm{~min}$, and FLAG-tagged proteins were captured by incubation

26 for 2 h with anti-FLAG M2 antibody (Sigma Aldrich) and Protein G Sepharose 4 Fast Flow (GE

27 Healthcare). After four washes of resin with CoIP buffer, bound proteins were eluted by incubation

28 at $95^{\circ} \mathrm{C}$ in lithium dodecyl sulfate-based gel loading buffer. 
For signaling assays, 3T3-[Shh-BlastR;Cas9] cells transduced with gRNAs as indicated

2 were seeded in a 24-well plate and grown until confluency. Growth medium was then replaced

3 with serum starvation medium with or without ShhN conditioned medium at the appropriate

4 dilution. After $24 \mathrm{~h}$, cells were lysed in SDS sample buffer (50 mM Tris HCl pH 6.8, 8\% v/v

5 glycerol, 2\% w/v SDS, $100 \mathrm{mM}$ DTT, $0.1 \mathrm{mg} / \mathrm{mL}$ bromophenol blue), boiled and sonicated.

6 Samples were loaded onto a 4-15\% Criterion TGX Stainfree gel (Bio-Rad), and run for 25 min,

$7300 \mathrm{~V}$ in Tris/Glycine/SDS buffer (Bio-Rad), before being transferred onto a PVDF membrane

8 using a Transblot Turbo system (Bio-Rad). Membranes were blocked in 1:1 PBS:SeaBlock

9 (Thermo Scientific) for $1 \mathrm{~h}$ at room temperature, and subsequently incubated with the indicated

10 primary antibody for $16 \mathrm{~h}$ at $4{ }^{\circ} \mathrm{C}$ (primary antibodies to GLI1, GLI2, GLI3, SUFU, and importin-

$11 \beta$, as described in Supplementary Table 8). Membranes were washed, incubated with HRP-

12 conjugated secondary antibody, washed again, developed using Supersignal West Femto

13 Maximum Sensitivity Substrate (Thermo Fisher) and imaged on a ChemiDoc MP (Bio-Rad).

14 Membranes were stripped using Restore Western Blot stripping buffer (Thermo-Fisher) and re15 probed as described above.

16 For analysis of immunoprecipitations, Western blotting was performed as described above,

17 except samples were separated in 4-12\% Bis-Tris PAGE gels (Invitrogen) using MOPS running

18 buffer, transferred to PVDF membranes using the Criterion Blotter system (Bio-Rad), developed

19 using ECL or EGL 2 chemiluminescence detection kits (Pierce), and imaged on a Chemidoc Touch

20 system (Bio-Rad).

Large-scale affinity purification and mass spectrometry

Affinity purifications were conducted as described ${ }^{69}$. Briefly, approximately 500-1000 $\mu \mathrm{l}$

24 packed cell volume was lysed in LAP purification buffer containing 0.3\% NP-40. Lysate was

25 cleared sequentially at 16,000 $\mathrm{x} g$ and 100,000 $\mathrm{x} g$ before incubation with anti-GFP antibody

26 coupled to Protein A resin. After protein capture and washes, bound LAP-tagged proteins were

27 eluted by incubation with HRV3C protease. For mass spectrometry analysis 'A' (see

28 Supplementary Table 6), eluted proteins were further purified by capture on S-Protein agarose

29 followed by elution at $95^{\circ} \mathrm{C}$ in lithium dodecyl sulfate-based gel loading buffer.

For protein analysis by mass spectrometry, gel slices containing affinity-purified proteins

31 were washed with $50 \mathrm{mM}$ ammonium bicarbonate, followed by reduction with DTT ( $5 \mathrm{mM}$ ) and 
1 alkylation using propionamide $(10 \mathrm{mM})$. Gel slices were further washed with an acetonitrile-

2 ammonium bicarbonate buffer until all stain was removed. $120 \mathrm{ng}$ of Trypsin/LysC (Promega)

3 reconstituted in $0.1 \%$ ProteaseMAX (Promega) with $50 \mathrm{mM}$ ammonium bicarbonate was added

4 to each gel band; after $30 \mathrm{~min} \sim 20 \mu \mathrm{L}$ of additional $50 \mathrm{mM}$ ammonium bicarbonate in $0.1 \%$

5 ProteaseMAX was added. Digestion was then allowed to occur overnight at $37^{\circ} \mathrm{C}$. Peptides were

6 extracted from the gels in duplicate followed by drying using a SpeedVac concentrator. Peptide

7 pools were then reconstituted and injected onto a C18 reversed phase analytical column, $\sim 20 \mathrm{~cm}$

8 in length, pulled and packed in-house. The UPLC was a NanoAcquity or M-Class column

9 (Waters), operated at $450 \mathrm{~nL} / \mathrm{min}$ using a linear gradient from 4\% mobile phase $\mathrm{B}$ to $45 \% \mathrm{~B}$.

10 Mobile phase A consisted of $0.2 \%$ formic acid, water, Mobile phase $\mathrm{B}$ was $0.2 \%$ acetic acid,

11 acetonitrile. The mass spectrometer was an Orbitrap Elite or Fusion (Thermo Fisher) set to acquire

12 in a data-dependent fashion selecting and fragmenting the 15 most intense precursor ions in the

13 ion-trap, where the exclusion window was set at 45 seconds and multiple charge states of the same

14 ion were allowed.

\section{QUANTIFICATION AND STATISTICAL ANALYSIS}

17 Analysis of CRISPR-based screens

CRISPR-based screens were analyzed as described ${ }^{27}$, processing data from each screen

batch separately. Briefly, to determine sgRNA counts in each sample, raw sequencing reads were trimmed to the 3'-most 17 nt of each protospacer and aligned to expected sgRNA sequences. This

21 alignment was carried out with the makeCounts script of the casTLE software package, which uses

22 Bowtie $^{73}$ to perform alignment with zero mismatches tolerated. The analyzeCounts script (v0.7 23 and v1.0) of the casTLE software available at https://bitbucket.org/dmorgens/castle ${ }^{27}$ was then

24 used to identify genes exhibiting significant enrichment or depletion and to estimate the phenotypic

25 effect size for each gene. This method uses an empirical Bayesian approach to score genes

26 according to the log-likelihood ratio that a gene's observed changes in sgRNA counts is drawn

27 from a model of gene effect versus the distribution of negative control sgRNAs. An expected

28 negative score distribution is obtained by random permutation of gene-targeting sgRNA fold-

29 change values and used to determine a $P$ value for each gene. Note that the use of 100,000

30 permutations leads to a minimum reported $P$ value of $1 \times 10^{-5}$. 
Genes targeted by our sgRNA library that lacked an NCBI identifier or that severely

2 affected growth (casTLE effect size $\leq-2.5$ and casTLE $P$ value $<0.005$ ) were not considered for

3 further analysis but are included in Supplementary Table 3. Negative and positive reference genes

4 were defined for growth and signaling phenotypes based on gene sets previously defined by Hart

5 et al. ${ }^{28}$ and Roosing et al. ${ }^{9}$, respectively (Supplementary Table 3). Precision-recall and ROG curves

6 using these reference genes were computed in Matlab (Mathworks). The hit genes at 10\% and 20\%

7 false discovery rate cutoffs were defined using the precision-recall threshold values at precision of

80.9 and 0.8 , yielding $P$ value cutoffs of 0.0163 and 0.0338 , respectively. Ciliopathy-associated

9 genes were defined from the OMIM website. Functional category enrichment analysis for hit genes

10 in the 10\% FDR category was performed using the DAVID website's Functional Annotation Chart

11 tool using all mouse genes as the background ${ }^{29}$. A second analysis was also performed using human

12 homologs of the top hits using all human genes as the background. Significance of overlap between

13 the top 15 congenital heart defect genes reported by Sifrim et al. ${ }^{49}$ and $P$ values from functional

14 screening was assessed using the Kolmogorov-Smirnov test.

Quantification of Hh signaling assays

17 Blasticidin-based inhibition of cell growth was determined by normalizing raw CellTiter-

18 Blue fluorescence such that growth in the absence of blasticidin corresponds to $100 \%$ growth while

19 medium alone corresponds to $\%$ growth. The $\mathrm{IC}_{50}$ for blasticidin was determined using Prism 7.0

20 (Graphpad Software) to fit dose-response data to $\log$ (inhibitor) versus response with variable slope.

21 Data shown are average of two to five independent assays conducted in duplicate for each 22 condition and cell line.

23 Dual-luciferase data were analyzed by first subtracting background signal such that cells

24 without luciferase give readings of zero. Firefly to Renilla (8x-Gli to constitutive) ratios were then

25 calculated and normalized such that unstimulated wildtype cells have a value equal to 1. Data

26 shown are average of replicate samples from a single experiment representative of 2-3 independent

27 repeats.

Quantification offluorescence microscopy images

Microscopy images were analyzed using Fiji ImageJ software (National Institutes of Health)

31 and a custom Matlab (Mathworks) script. Local background subtraction was performed on all 
1 images before analysis. To determine ciliary frequency, cells were manually scored for the presence

2 or absence of a cilium using ARL13B and acetylated tubulin as ciliary markers. For ciliary length

3 and intensity of ciliary markers analyses, the ARL13B and/or acetylated tubulin channels were

4 used to create a ciliary mask and to measure cilium length. The ciliary mask was then used to

5 identify and measure ciliary signal in the other channels. The $\gamma$-tubulin or ninein signal (staining

6 centrioles) was used to orient all cilia from base to tip. Tip fluorescence for GLI2 and GLI3 was

7 defined as the summed fluorescence in the final five pixels of each cilium, regardless of length. For

8 ARMC9-FLAG localization, the ciliary fluorescence of each individual cilium was normalized to

9 1, and each axoneme divided in 20 equal distance bins. The fraction of FLAG signal within each

10 bin is plotted for $\mathrm{N}>500$ cilia from one out of three representative experiments. Where indicated

11 in the Figure Legend, data is shown relative to values obtained for the control sgRNA using Prism

127 (GraphPad Software).

13 Differences in cilium length distribution were tested for significance using the Kolmogorov-

14 Smirnov test in Matlab. Line plots of fluorescence intensity along cilia were generated in ImageJ.

15 Centriole counting measurements were done manually, using the $\gamma$-tubulin and centrin3

16 staining to guide centriole calling. Cell cycle stage was determined using the DNA morphology,

17 and statistical significance was determined using Fisher's exact test. Cell counts for each of the five

18 centriole number categories were used for statistical comparisons between genotypes. For

19 comparisons between cell cycle stages, cells observed to have 2 or 3-4 centrioles were grouped into

20 one category to account for the expected reduction in centriole number upon completion of

21 mitosis.

23 Analysis of mass spectrometry data

24 MS/MS data were analyzed using both Preview and Byonic v2.10.5 (ProteinMetrics). All

25 data were first analyzed in Preview to provide recalibration criteria if necessary and then

26 reformatted to MGF format before full analysis with Byonic. Data were searched at 12 ppm mass

27 tolerances for precursors, with 0.4 Da fragment mass tolerances assuming up to two missed

28 cleavages and allowing for fully specific and ragged tryptic peptides. The database used was

29 Uniprot for Mus musculus downloaded on 10/25/2016. These data were validated at a 1\% false

30 discovery rate using typical reverse-decoy techniques ${ }^{74}$. The resulting identified peptide spectral 
1 matches and assigned proteins were then exported for further analysis using MatLab (MathWorks)

2 to provide visualization and statistical characterization.

4 Analysis of CRISPR growth screen datasets

5 Gene-level growth phenotype data ${ }^{61}$ were downloaded from the Achilles website.

6 Hierarchical clustering using uncentered correlation and average linkage settings was performed

7 using Cluster 3.0 software ${ }^{75}$, and clustered data were visualized in Java Treeview ${ }^{76}$.

$9 \quad$ Phylogenetic analysis

10 Homologs for Tubd 1 and Tubel were either previously described ${ }^{64}$ or identified using 11 protein BLAST. Homologs for Ted1 and Ted2 were identified using iterative searches with PSI-

12 BLAST ${ }^{77}$. To analyze sequence divergence, homolog sequences were first aligned using Clustal

13 Omega $^{78}$. Phylogenetic trees were generated via neighbor joining with distance correction using

14 Simple Phylogeny ${ }^{78}$ and visualized using Unrooted $^{79}$.

\section{DATA AND SOFTWARE AVAILABILITY}

17 Data resources including aligned sgRNA sequences, screen sequencing data, casTLE 18 output, positive and negative reference gene sets for growth and ciliary signaling phenotypes, 19 functional categories enriched among hit genes, phylogenetic analysis of TED complex 20 components, and mass spectrometry-based protein identifications can be found in Supplementary

21 Tables 2, 3, 5, and 6. Software used for casTLE analysis can be found at 22 http://bitbucket.org/dmorgens/castle. Scripts for quantification of cilium intensities and length 23 are available upon request. 


\section{References}

1. Goetz, S.C. \& Anderson, K.V. The primary cilium: a signalling centre during vertebrate development. Nat Rev Genet 11,331-44 (2010).

2. Nachury, M.V. How do cilia organize signalling cascades? Philos Trans R Soc Lond B Biol Sci 369, 20130465-20130465 (2014).

3. Bangs, F. \& Anderson, K.V. Primary Cilia and Mammalian Hedgehog Signaling. Cold Spring Harb Perspect Biol $\mathbf{9}$ (2017).

4. Braun, D.A. \& Hildebrandt, F. Ciliopathies. Cold Spring Harb Perspect Biol 9, a028191 (2017).

5. Akizu, N. et al. Mutations in CSPP1 lead to classical Joubert syndrome. Am 7 Hum Genet 94, 80-6 (2014).

6. Briscoe, J. \& Therond, P.P. The mechanisms of Hedgehog signalling and its roles in development and disease. Nat Rev Mol Cell Biol 14, 416-29 (2013).

7. Wu, F., Zhang, Y., Sun, B., McMahon, A.P. \& Wang, Y. Hedgehog Signaling: From Basic Biology to Cancer Therapy. Cell Chem Biol 24, 252-280 (2017).

8. Pak, E. \& Segal, R.A. Hedgehog Signal Transduction: Key Players, Oncogenic Drivers, and Cancer Therapy. Dev Cell 38, 333-44 (2016).

9. Roosing, S. et al. Functional genome-wide siRNA screen identifies KIAA0586 as mutated in Joubert syndrome. Elife 4, e06602 (2015).

10. Wheway, G. et al. An siRNA-based functional genomics screen for the identification of regulators of ciliogenesis and ciliopathy genes. Nat Cell Biol 17, 1074-87 (2015).

11. Jacob, L.S. et al. Genome-wide RNAi screen reveals disease-associated genes that are common to Hedgehog and Wnt signaling. Sci Signal 4, ra4 (2011).

12. Kim, J. et al. Functional genomic screen for modulators of ciliogenesis and cilium length. Nature 464, 1048-51 (2010).

13. Shalem, O. et al. Genome-scale CRISPR-Cas9 knockout screening in human cells. Science 343, 84-7 (2014).

14. Koike-Yusa, H., Li, Y., Tan, E.P., Velasco-Herrera Mdel, G. \& Yusa, K. Genome-wide recessive genetic screening in mammalian cells with a lentiviral CRISPR-guide RNA library. Nat Biotechnol 32, 267-73 (2014).

15. Wang, T., Wei, J.J., Sabatini, D.M. \& Lander, E.S. Genetic screens in human cells using the CRISPR-Cas9 system. Science 343, 80-4 (2014). 
16. Zhou, Y. et al. High-throughput screening of a CRISPR/Cas9 library for functional genomics in human cells. Nature 509, 487-91 (2014).

17. Deans, R.M. et al. Parallel shRNA and CRISPR-Cas9 screens enable antiviral drug target identification. Nat Chem Biol 12, 361-6 (2016).

18. Wang, T. et al. Identification and characterization of essential genes in the human genome. Science 350, 1096-101 (2015).

19. Wang, T. et al. Gene Essentiality Profiling Reveals Gene Networks and Synthetic Lethal Interactions with Oncogenic Ras. Cell 168, 890-903 e15 (2017).

20. Hart, T. et al. High-Resolution CRISPR Screens Reveal Fitness Genes and GenotypeSpecific Cancer Liabilities. Cell 163, 1515-1526 (2015).

21. Tzelepis, K. et al. A GRISPR Dropout Screen Identifies Genetic Vulnerabilities and Therapeutic Targets in Acute Myeloid Leukemia. Cell Rep 17, 1193-1205 (2016).

22. Orchard, R.G. et al. Discovery of a proteinaceous cellular receptor for a norovirus. Science 353, 933-6 (2016).

23. Park, R.J. et al. A genome-wide CRISPR screen identifies a restricted set of HIV host dependency factors. Nat Genet 49, 193-203 (2017).

24. Arroyo, J.D. et al. A Genome-wide CRISPR Death Screen Identifies Genes Essential for Oxidative Phosphorylation. Cell Metab 24, 875-885 (2016).

25. Taipale, J. et al. Effects of oncogenic mutations in Smoothened and Patched can be reversed by cyclopamine. Nature 406, 1005-9 (2000).

26. Morgens, D.W. et al. Genome-scale measurement of off-target activity using Cas9 toxicity in high-throughput screens. Nat Commun 8, 15178 (2017).

27. Morgens, D.W., Deans, R.M., Li, A. \& Bassik, M.C. Systematic comparison of CRISPR/Cas9 and RNAi screens for essential genes. Nat Biotechnol 34, 634-6 (2016).

28. Hart, T., Brown, K.R., Sircoulomb, F., Rottapel, R. \& Moffat, J. Measuring error rates in genomic perturbation screens: gold standards for human functional genomics. Mol Syst Biol 10, 733 (2014).

29. Huang, D.W., Sherman, B.T. \& Lempicki, R.A. Systematic and integrative analysis of large gene lists using DAVID bioinformatics resources. Nat Protoc 4, 44-57 (2009).

30. Webb, T.R. et al. Diphthamide modification of eEF2 requires a J-domain protein and is essential for normal development. 7 Cell Sci 121, 3140-5 (2008).

31. Chen, C.M. \& Behringer, R.R. Ovcal regulates cell proliferation, embryonic development, and tumorigenesis. Genes Dev 18, 320-32 (2004). 
32. Loucks, C.M. et al. Matching two independent cohorts validates DPH1 as a gene responsible for autosomal recessive intellectual disability with short stature, craniofacial, and ectodermal anomalies. Hum Mutat 36, 1015-1019 (2015).

33. Allen, B.L. et al. Overlapping roles and collective requirement for the coreceptors GAS1, CDO, and BOG in SHH pathway function. Dev Cell 20, 775-87 (2011).

34. Izzi, L. et al. Boc and Gas 1 each form distinct Shh receptor complexes with Ptch1 and are required for Shh-mediated cell proliferation. Dev Cell 20, 788-801 (2011).

35. Lee, Y.L. et al. Cbyl promotes Ahil recruitment to a ring-shaped domain at the centriolecilium interface and facilitates proper cilium formation and function. Mol Biol Cell 25, 2919-33 (2014).

36. Burke, M.C. et al. Chibby promotes ciliary vesicle formation and basal body docking during airway cell differentiation. The Journal of cell biology 207, 123-137 (2014).

37. Glazer, A.M. et al. The Zn finger protein Iguana impacts Hedgehog signaling by promoting ciliogenesis. Dev Biol 337, 148-56 (2010).

38. Li, F.Q. et al. BAR Domain-Containing FAM92 Proteins Interact with Chibby1 To Facilitate Ciliogenesis. Mol Cell Biol 36, 2668-2680 (2016).

39. Dorn, K.V., Hughes, C.E. \& Rohatgi, R. A Smoothened-Evc2 complex transduces the Hedgehog signal at primary cilia. Dev Cell 23, 823-35 (2012).

40. Pusapati, G.V. et al. EFCAB7 and IQCE regulate hedgehog signaling by tethering the EVG-EVC2 complex to the base of primary cilia. Dev Cell 28, 483-96 (2014).

41. Ruiz-Perez, V.L. \& Goodship, J.A. Ellis-van Creveld syndrome and Weyers acrodental dysostosis are caused by cilia-mediated diminished response to hedgehog ligands. Am $\mathcal{J}$ Med Genet C Semin Med Genet 151C, $341-51$ (2009).

42. Shaheen, R. et al. Genomic analysis of Meckel-Gruber syndrome in Arabs reveals marked genetic heterogeneity and novel candidate genes. Eur 7 Hum Genet 21, 762-8 (2013).

43. Shaheen, R. et al. Characterizing the morbid genome of ciliopathies. Genome Biol 17, 242 (2016).

44. Kar, A., Phadke, S.R., Das Bhowmik, A. \& Dalal, A. Whole exome sequencing reveals a mutation in ARMC9 as a cause of mental retardation, ptosis and polydactyly. bioRxiv, 109124 (2017).

45. Panigrahi, I., Phadke, S.R. \& Agarwal, S.S. Mental retardation, ptosis and polydactyly: a new autosomal recessive syndrome? Clin Dysmorphol 11, 289-92 (2002).

46. Nager, A.R. et al. An Actin Network Dispatches Ciliary GPGRs into Extracellular Vesicles to Modulate Signaling. Cell 168, 252-263.e14 (2017). 
47. $\mathrm{Xu}, \mathrm{M}$. et al. Mutations in the Spliceosome Component CWC27 Cause Retinal Degeneration with or without Additional Developmental Anomalies. Am 7 Hum Genet 100, 592-604 (2017).

48. Oegema, R. et al. Human mutations in integrator complex subunits link transcriptome integrity to brain development. PLoS Genet 13, e1006809 (2017).

49. Sifrim, A. et al. Distinct genetic architectures for syndromic and nonsyndromic congenital heart defects identified by exome sequencing. Nat Genet 48, 1060-5 (2016).

50. Sollis, E. et al. Identification and functional characterization of de novo FOXP1 variants provides novel insights into the etiology of neurodevelopmental disorder. Hum Mol Genet 25, 546-57 (2016).

51. Li, Y. et al. Global genetic analysis in mice unveils central role for cilia in congenital heart disease. Nature 521, 520-4 (2015).

52. Chang, P. \& Stearns, T. Delta-tubulin and epsilon-tubulin: two new human centrosomal tubulins reveal new aspects of centrosome structure and function. Nat Cell Biol 2, 30-5 (2000).

53. Dupuis-Williams, P. et al. Functional role of epsilon-tubulin in the assembly of the centriolar microtubule scaffold. F Cell Biol 158, 1183-93 (2002).

54. Dutcher, S.K., Morrissette, N.S., Preble, A.M., Rackley, C. \& Stanga, J. Epsilon-tubulin is an essential component of the centriole. Mol Biol Cell 13, 3859-69 (2002).

55. O'Toole, E.T., Giddings, T.H., McIntosh, J.R. \& Dutcher, S.K. Three-dimensional organization of basal bodies from wild-type and delta-tubulin deletion strains of Chlamydomonas reinhardtii. Mol Biol Cell 14, 2999-3012 (2003).

56. Ross, I., Clarissa, C., Giddings, T.H., Jr. \& Winey, M. epsilon-tubulin is essential in Tetrahymena thermophila for the assembly and stability of basal bodies. 7 Cell Sci 126, 3441-51 (2013).

57. Sharma, A. et al. Centriolar CPAP/SAS-4 Imparts Slow Processive Microtubule Growth. Dev Cell 37, 362-76 (2016).

58. Schmidt, T.I. et al. Control of centriole length by CPAP and CP110. Curr Biol 19, 100511 (2009).

59. Kirkham, M., Muller-Reichert, T., Oegema, K., Grill, S. \& Hyman, A.A. SAS-4 is a C. elegans centriolar protein that controls centrosome size. Cell 112, 575-87 (2003).

60. Wong, Y.L. et al. Cell biology. Reversible centriole depletion with an inhibitor of Polo-like kinase 4. Science 348, 1155-60 (2015). 
61. Aguirre, A.J. et al. Genomic Copy Number Dictates a Gene-Independent Cell Response to CRISPR/Cas9 Targeting. Cancer Discov 6, 914-29 (2016).

62. Chaki, M. et al. Exome Capture Reveals ZNF423 and CEP164 Mutations, Linking Renal Ciliopathies to DNA Damage Response Signaling. Cell 150, 533-548 (2012).

63. MacArthur, D.G. et al. Guidelines for investigating causality of sequence variants in human disease. Nature 508, 469-76 (2014).

64. Turk, E. et al. Zeta-Tubulin Is a Member of a Conserved Tubulin Module and Is a Component of the Centriolar Basal Foot in Multiciliated Cells. Curr Biol 25, 2177-83 (2015).

65. Pimenta-Marques, A. et al. A mechanism for the elimination of the female gamete centrosome in Drosophila melanogaster. Science 353, aaf4866 (2016).

66. Zheng, X. et al. Molecular basis for CPAP-tubulin interaction in controlling centriolar and ciliary length. Nat Commun 7, 11874 (2016).

67. Bond, J. et al. A centrosomal mechanism involving CDK5RAP2 and CENPJ controls brain size. Nat Genet 37, 353-5 (2005).

68. Gilmore, E.C. \& Walsh, C.A. Genetic causes of microcephaly and lessons for neuronal development. Wiley Interdiscip Rev Dev Biol 2, 461-78 (2013).

69. Liew, G.M. et al. The intraflagellar transport protein IFT27 promotes BBSome exit from cilia through the GTPase ARL6/BBS3. Dev Cell 31, 265-78 (2014).

70. Frank-Kamenetsky, M. et al. Small-molecule modulators of Hedgehog signaling: identification and characterization of Smoothened agonists and antagonists. $\mathcal{F}$ Biol 1, 10 (2002).

71. Chen, J.K., Taipale, J., Young, K.E., Maiti, T. \& Beachy, P.A. Small molecule modulation of Smoothened activity. Proc Natl Acad Sci U SA 99, 1407 1-6 (2002).

72. Breslow, D.K., Koslover, E.F., Seydel, F., Spakowitz, A.J. \& Nachury, M.V. An in vitro assay for entry into cilia reveals unique properties of the soluble diffusion barrier. 7 Cell Biol 203, 129-47 (2013).

73. Langmead, B., Trapnell, G., Pop, M. \& Salzberg, S.L. Ultrafast and memory-efficient alignment of short DNA sequences to the human genome. Genome Biol 10, R25 (2009).

74. Elias, J.E. \& Gygi, S.P. Target-decoy search strategy for increased confidence in largescale protein identifications by mass spectrometry. Nat Methods 4, 207-14 (2007).

75. de Hoon, M.J., Imoto, S., Nolan, J. \& Miyano, S. Open source clustering software. Bioinformatics 20, 1453-4 (2004). 
76. Saldanha, A.J. Java Treeview--extensible visualization of microarray data. Bioinformatics 20, 3246-8 (2004).

77. Altschul, S.F. et al. Gapped BLAST and PSI-BLAST: a new generation of protein database search programs. Nucleic Acids Res 25, 3389-402 (1997).

78. Li, W. et al. The EMBL-EBI bioinformatics web and programmatic tools framework. Nucleic Acids Res 43, W580-4 (2015).

79. Perrière, G. \& Gouy, M. WWW-query: an on-line retrieval system for biological sequence banks. Biochimie 78, 364-369 (1996). 


\section{Figure Legends}

2 Figure 1. Development of a Hedgehog pathway reporter-based screening strategy

3 a) A transcriptional reporter attaches 8 copies of the GLI binding sequence (Gli-BS) to a minimal

4 promoter $\left(\mathrm{P}_{\mathrm{min}}\right)$ to convert $\mathrm{Hh}$ signals into blasticidin resistance. b) Blasticidin resistance was

5 assayed across a range of concentrations in stimulated $(+\mathrm{ShhN})$ and unstimulated 3T3-[Shh-

6 BlastR;Cas9] cells. c) Overview of the Hh pathway, with key negative and positive regulators

7 shown in red and green, respectively (left). Effects of control sgRNAs on blasticidin resistance in

8 stimulated and unstimulated 3T3-[Shh-BlastR;Cas9] cells (right). Mean inhibitory concentration

$950\left(\mathrm{IC}_{50}\right)$ values and standard deviations are shown. $\mathrm{N}=2$ to 5 independent experiments, each

10 performed in duplicate. d) Overview of the screening strategy. Cells receiving a negative control

11 (Ctrl) sgRNA, a positive regulator-targeting sgRNA, and a negative regulator-targeting sgRNA are

12 shaded grey, green, and red, respectively. e) Flow cytometry histograms of cell mixtures showing

13 the fraction of GFP positive (Smo sgRNA-2, green) cells either in the absence of selection (left) or

14 after two rounds of signaling and selection (right). Cells expressing Smo-targeting sgRNA-2 are

15 specifically depleted in the signaling/selection conditions. f) Quantification of cell depletion as in $16(\mathbf{e})$.

Figure 2. Overview of genome-wide screen results

19 a) Scatter plot showing $\log _{2}$ of normalized sgRNA counts in selected versus unselected cell pools, with sgRNAs targeting different genes shown in indicated colors. b) Volcano plot of casTLE $P$

21 values against effect sizes for all genes (after filtering; see Methods), with core Hh pathway

22 components in red. Green and yellow areas indicate $P$-value cutoffs corresponding to $10 \%$ and $2320 \%$ false discovery rate, respectively, with the number of genes in each area indicated. c)

24 Schematic illustration of a primary cilium, with known structural features and associated hit genes 25 shown. Newly identified hit genes are in red font. For the IFT-A and IFT-B complexes, genes 26 detected as hits are shown in red and blue boxes, respectively, while non-hit genes are in grey 27 boxes. d) GO terms enriched among the top 472 hit genes. Bars indicate $P$-values from DAVID 28 gene enrichment analysis; numbers above bars indicate number of hit genes for each class. 
1 a) Assessment of screen performance using positive and negative reference genes, as determined

2 by precision-recall analysis (left) and ROC curve (right), with the area under each curve (AUC)

3 shown. Dashed lines indicate performance of a random classification model. b) Analysis of hit gene

4 detection for select gene categories, with the fraction of hits detected at $10 \%$ or 20\% FDR, not

5 detected, or not determined shown; see Supplementary Table 3 for details. The NPHP category

6 includes genes mutated exclusively in NPHP and not other ciliopathies. Abbreviations: SRTD

7 (short rib thoracic dysplasia), JATD (Jeune asphyxiating thoracic dysplasia), OFD (Oral-Facial-

8 Digital Syndrome). c) Hit gene identification is compared for the indicated datasets. Pie charts

9 show the fraction of genes detected as hits across all genes included in part (b), except the NPHP-

10 specific category; see Supplementary Figure 4 for detail among individual categories.

12 Figure 4. Characterization of FAM92A and TTC23 as transition zone and EvG zone 13 components

14 a) A luciferase-based Hh pathway reporter assay was performed for cells transduced with the 15 indicated sgRNAs and transfected with plasmids expressing either Fam92a-3xFLAG (Fam92a) or $16 G F P-F K B P(G F P)$. Cells were untreated (-SAG) or stimulated with the SMO agonist SAG (+SAG).

17 Mean and standard deviation for 4 replicate measurements from one of two representative 18 experiments. b) 3T3-[Shh-BlastR;Cas9] cells transduced with Fam92a-targeting sgRNAs are 19 deficient in cilium assembly, as visualized by immunofluorescent staining of ARL13B and 20 acetylated tubulin; centrioles are marked by $\gamma$-tubulin. Bars show mean fraction of ciliated cells; 21 dots show ciliated fraction in each independent experiment ( $>400$ cells were analyzed from two 22 independent experiments). Scale bar: $5 \mu \mathrm{m}$ c) Mass spectrometry analysis of FAM92A-associated 23 proteins purified from IMCD3 cells reveals transition zone components CBY1 and DZIP1L. For 24 each protein, the normalized spectral abundance factor (NSAF; number of spectra identified per 25100 amino acids), the percent of the protein covered by identified peptides, and the percentile rank 26 of the corresponding gene in the screen dataset are indicated. d) FAM92A (green) localizes to the 27 transition zone of IMCD3 cells, distal to centrioles marked by $\gamma$-tubulin (magenta) and proximal 28 to the ciliary shaft marked by ARL13B (red). Scale bars: $5 \mu \mathrm{m}$ and $1 \mu \mathrm{m}$ (insets). e) Mass 29 spectrometry analysis of TTC23-associated proteins purified from IMCD3 cells reveals EvC zone 30 components EFGAB7 and IQCE. Data are shown as in (c). f) TTC23-LAP stably expressed in 31 IMCD3 cells co-localizes with EvC zone component IQGE, distal to transition zone component 
1 FAM92A. Line plots show normalized intensity for the indicated markers along the length of the

2 cilium; tick marks are $1 \mu \mathrm{m}$ intervals. Scale bars: $5 \mu \mathrm{m}$ and $1 \mu \mathrm{m}$ (insets). g) Ciliary IQCE levels

3 were measured for cells transduced with control (Ctrl) sgRNA or sgRNAs targeting Ttc23. The

4 median and interquartile range are plotted for $\mathrm{N}>295$ cells measured in each condition from a

5 representative experiment out of two independent replicates. h) Knockdown of Iqce in IMCD3 cells

6 leads to reduced ciliary GFP (TTC23-LAP) and IQCE (top). The median fluorescence and

7 interquartile range are plotted for n>190 cilia from a single experiment out of two (IQCE) or four

8 (GFP) replicate experiments (bottom). Scale bars: $1 \mu \mathrm{m}$.

Figure 5. Insights into ciliopathies from previously uncharacterized screen hits

11 a) Diagram of TXNDC15, with the transmembrane domain (TMD), thioredoxin domain, and 5

12 amino acid deletion found in MKS patients indicated (top). Hh luciferase reporter assay for cells

13 transduced with the indicated sgRNAs and transfected with plasmids encoding either GFP-FKBP

14 (GFP), wildtype Txndc15 (Tx-WT), or mutant Txndc15 (Tx-mut). Cells were untreated or stimulated

15 with ShhN. Mean and standard error for 6 replicate measurements from one of two representative

16 experiments. b) Analysis of cilia in 3T3-[Shh-BlastR;Cas9] cells transduced with Txndc15-targeting

17 sgRNAs reveals many distorted cilia with abnormal morphology or length and decreased ARL13B

18 staining. Lower images show enlarged micrographs for cilia marked by boxes A and B. Bar graph

19 shows mean and standard deviation (three independent experiments, >700 cells counted total).

20 Scale bars: $5 \mu \mathrm{m}$ (upper images) and $1 \mu \mathrm{m}$ (lower images). See also Supplementary Figure 5a-b. c)

21 Analysis of cilia in 3T3-[Shh-BlastR;Cas9] cells transduced with Armc9-targeting sgRNAs reveals

22 shorter cilia and reduced staining for the ciliary marker acetylated tubulin. Representative

23 micrographs are shown at top; mean cilium length relative to control sgRNA and standard

24 deviation are shown at bottom (three independent experiments, $>300$ cilia analyzed per

25 experiment). Scale bars: $5 \mu \mathrm{m}$. See also Supplementary Figure 5c. d) Analysis of ARMC9-FLAG

26 localization along IMCD3 cilia following stimulation of Hh signaling. Representative micrographs

27 showing localization relative to centrioles, marked by ninein, and the cilium, marked by ARL13B.

28 Scale bar: $1 \mu \mathrm{m}$. e) Plots of ARMC-FLAG intensity along the length of the cilium from base

29 (position 0) to tip (position 1.0) are shown for IMCD3 cells treated as indicated. The mean and

30 standard deviation are plotted after normalizing the total intensity in each cilium to 1.0 ( $\mathrm{N}>500$

31 cilia, representative example from 3 independent experiments). f) Fluorescence intensity of GLI3 
1 at the cilium tip was measured for the indicated cells in the presence or absence of ShhN. Mean

2 intensity (relative to control sgRNA cells $+\mathrm{ShhN}$ ) and standard error of the mean are shown for

3 each of three experiments $(\mathrm{N}>250$ cilia in each experiment). See also Supplementary Fig. 5d. g)

4 Table showing select clinical features in a diverse set of ciliopathies and their observation in the

5 context of specific mutations and syndromes. Colors indicate high (red), moderate (orange) and

6 low (yellow) prevalence. The name of each syndrome is listed; Joubert encompasses Joubert

7 Syndrome and related disorders.

$9 \quad$ Figure 6. TED1 and TED2 form a tetrameric complex with $\delta$ - and $\varepsilon$-tubulins

a) IMCD3 cells stably expressing TED2-LAP were immunostained with antibodies to centrin2 and

$11 \gamma$-tubulin to visualize centrioles. Scale bar: $5 \mu \mathrm{m}(2 \mu \mathrm{m}$ for insets). b) Mass spectrometry analysis

12 of TED2-associated proteins purified from IMCD3 cells reveals TED1, $\varepsilon$-tubulin, $\delta$-tubulin in

13 nearly stoichiometric amounts, as well as $\alpha / \beta$-tubulin and CENPJ. For each protein, the

14 normalized spectral abundance factor (NSAF; number of spectra identified per 100 amino acids),

15 the percent of the protein covered by identified peptides, and the percentile rank of the

16 corresponding gene in the screen dataset are indicated. c) Binding of TED1 and TED2 was

17 assessed via co-immunoprecipitations performed in HEK293T cells transfected with the indicated

18 proteins. Recovered proteins were analyzed by Western blot.

\section{Figure 7. The TED complex is required for centriole stability}

21 a) Cells transduced with the indicated sgRNAs were stained with antibodies to ninein (centrioles)

22 and polyglutamylated tubulin (GT335, centrioles and cilia). Scale bar: $5 \mu \mathrm{m}$. b) Hierarchical

23 clustering of relative growth scores across the indicated cell lines reveals that TED1, TED2, and

24 TUBE1 share a similar pattern of relative fitness. Blue and red shading indicates decreased and

25 increased proliferation relative to the average behavior across all cell lines. c) For cells transduced

26 with the indicated sgRNAs, centrioles were visualized by staining with antibodies to centrin3 and

$27 \gamma$-tubulin. Insets show centrin3 staining in mitotic cells, marked by yellow arrowheads. Scale bars:

$285 \mu \mathrm{m}$ (2 $\mu \mathrm{m}$ for insets). See also Supplementary Figure 6d. d Centrioles marked by centrin3 and

$29 \gamma$-tubulin were counted in cells at the indicated cell cycle stages, with each cell categorized as having

30 either zero centrioles, some centrioles but fewer than expected for that stage in the cell cycle (two

31 or four), the expected two or four centrioles, or more than the expected number of centrioles. 

aCC-BY-NC 4.0 International license.

1 Statistically significant differences in centriole counts are shown for select conditions $\left(^{*}, P<1 \times 10^{-}\right.$

$2 \quad$ 6; **, $P<1 \times 10^{-10}$; ***, $P<1 \times 10^{-60}$, determined by Fisher's exact test; see Methods). 
1 List of Supplementary Items

2

3 Supplementary Table 1. List of select sgRNA sequences.

4

5 Supplementary Table 2. List of sgRNA counts by deep sequencing of screen cell

6 pools.

7

8 Supplementary Table 3. casTLE output (gene scores) for genome-wide screen.

Supplementary Table 4. Summary of screen data for select genes of interest.

12 Supplementary Table 5. List of functional categories enriched among screen hits.

14 Supplementary Table 6. List of proteins identified by mass spectrometry in affinity

15 purifications.

17 Supplementary Table 7. Glustered growth data from Aguirre et al.

19 Supplementary Table 8. List of primary antibodies.

21 Supplementary Table 9. List of oligonucleotides and recombinant DNA. 

aCC-BY-NC 4.0 International license.

\section{Figure 1}

a

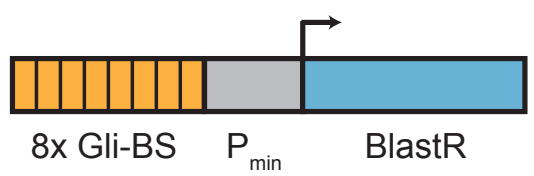

C

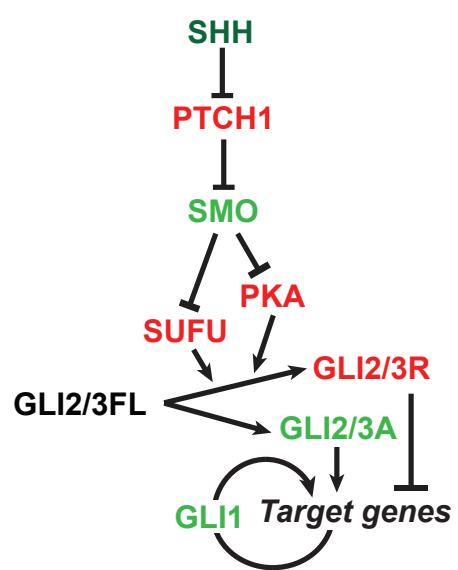

b

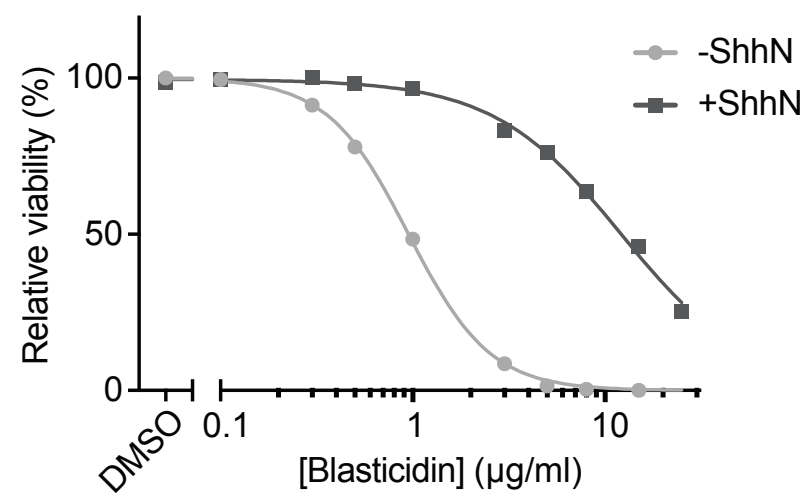

d sgRNA library (10 sgRNAs/gene)
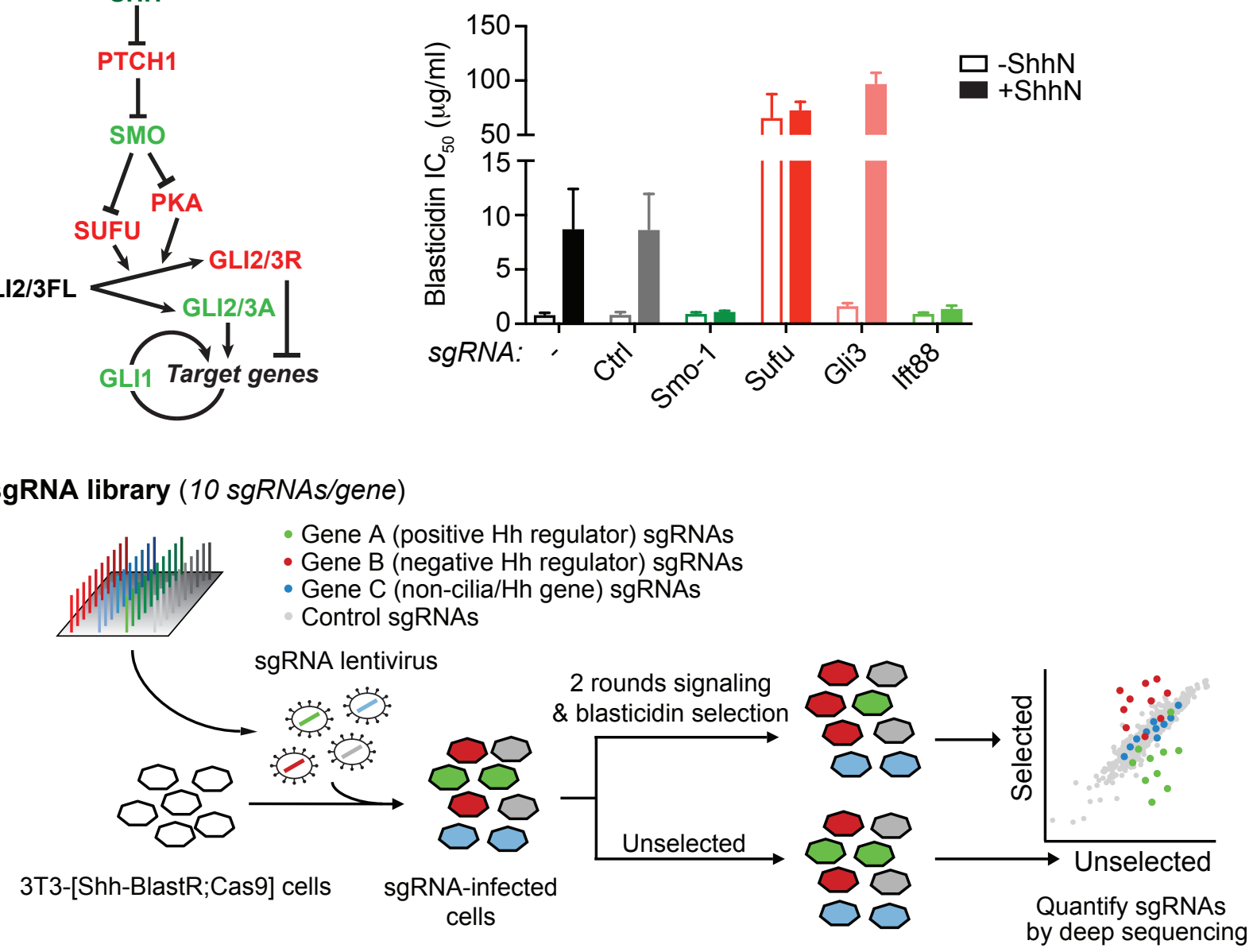

e
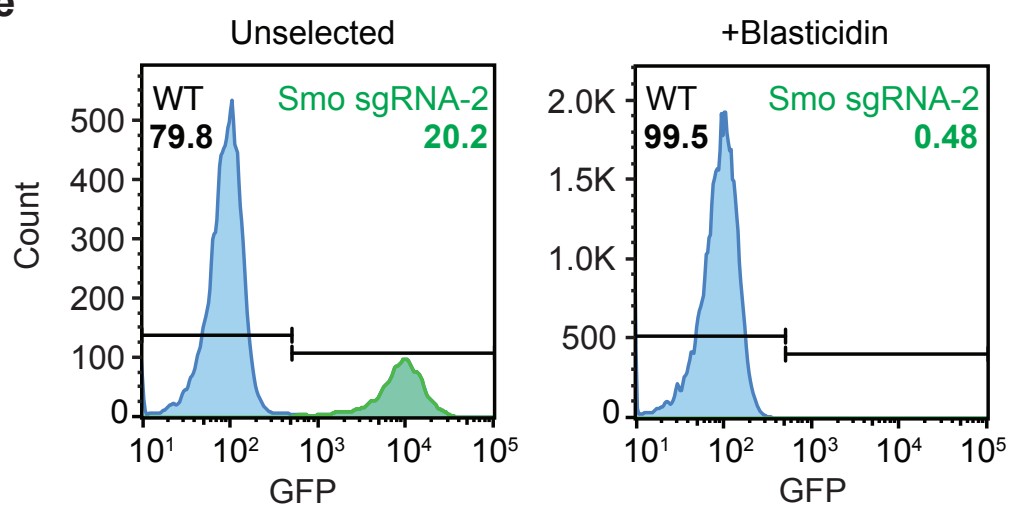

f

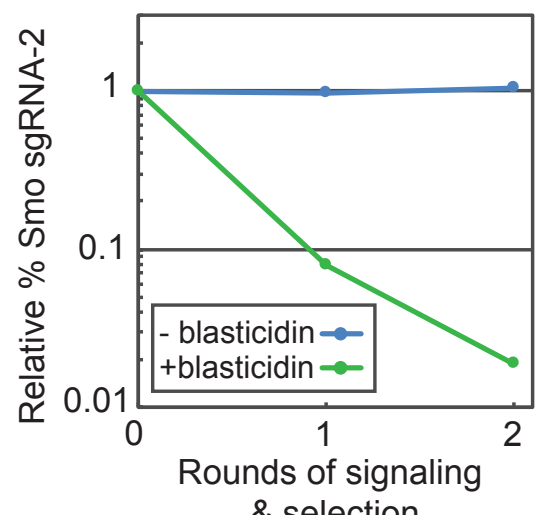

\& selection 
bioRxiv preprint doi: https://doi.org/10.1101/156059; this version posted June 27 2017. The copyright holder for this preprint (which was not certified by peer review) is the author/funder, who has granted bioRxiv a license to display the preprint in perpetuity. It is made available under aCC-BY-NC 4.0 International license.

\section{Figure 2}
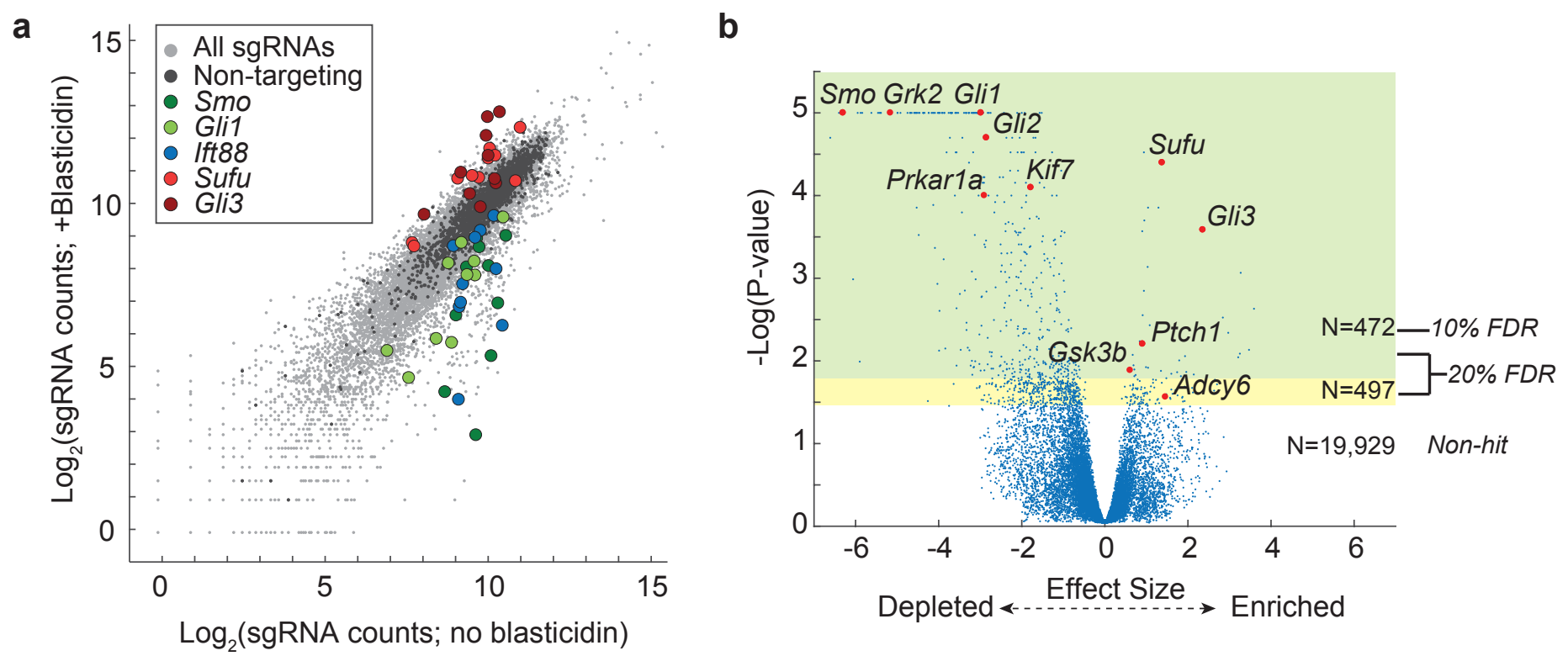

C

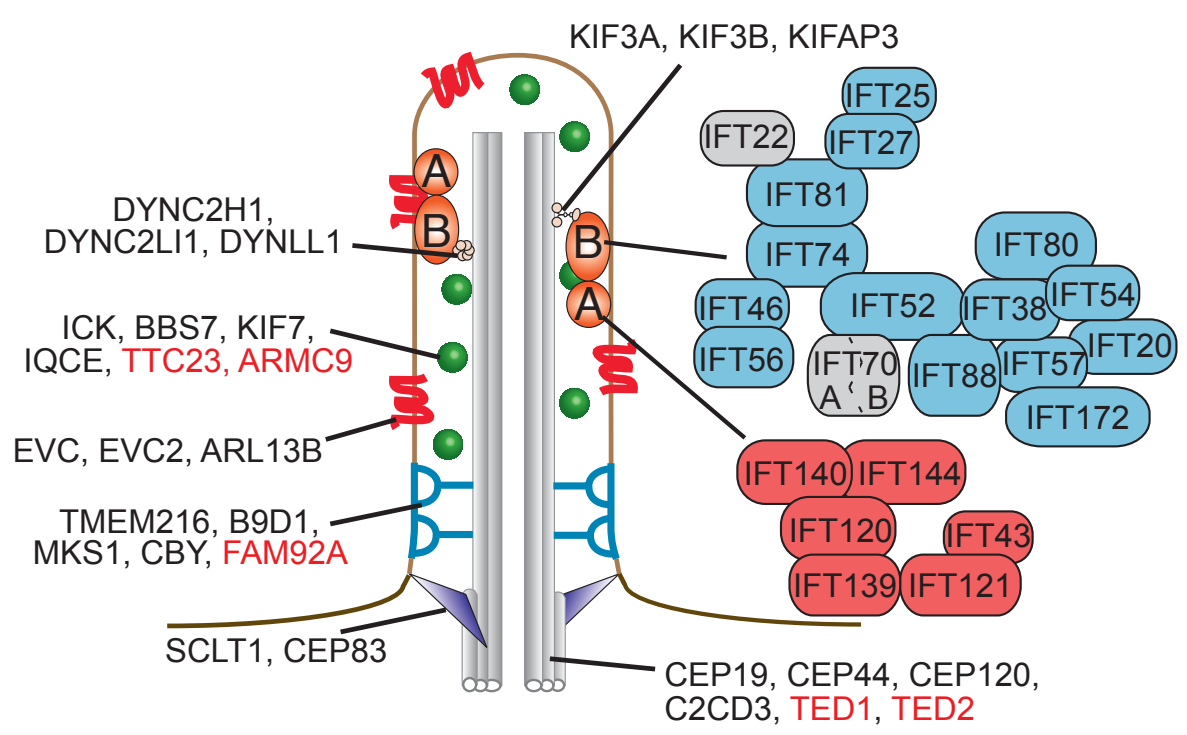

d

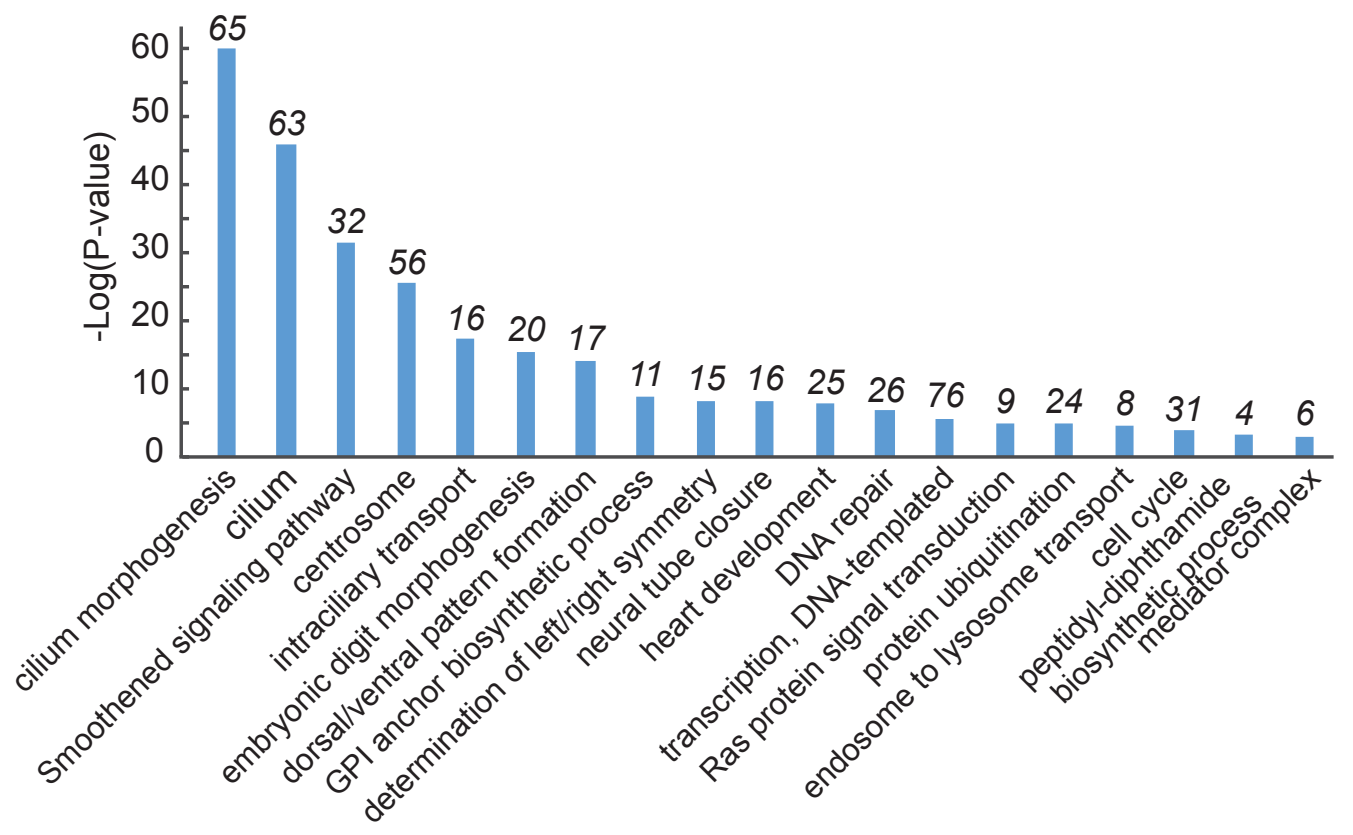




\section{Figure 3}

a

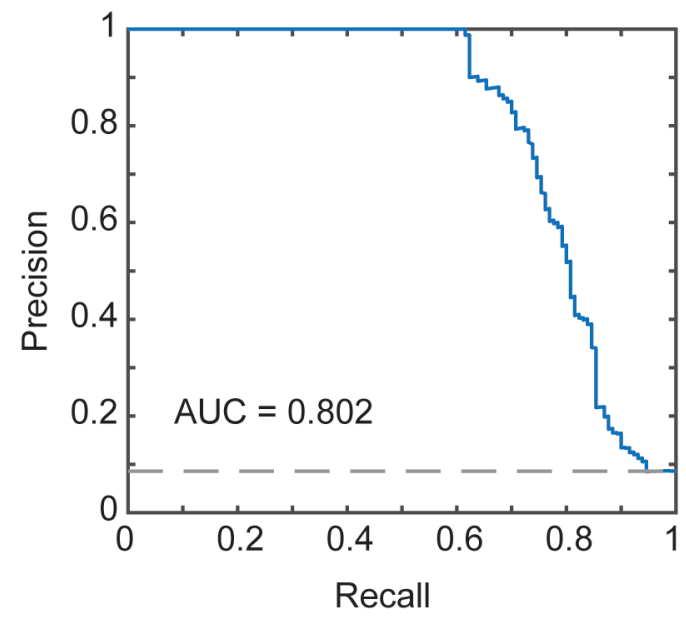

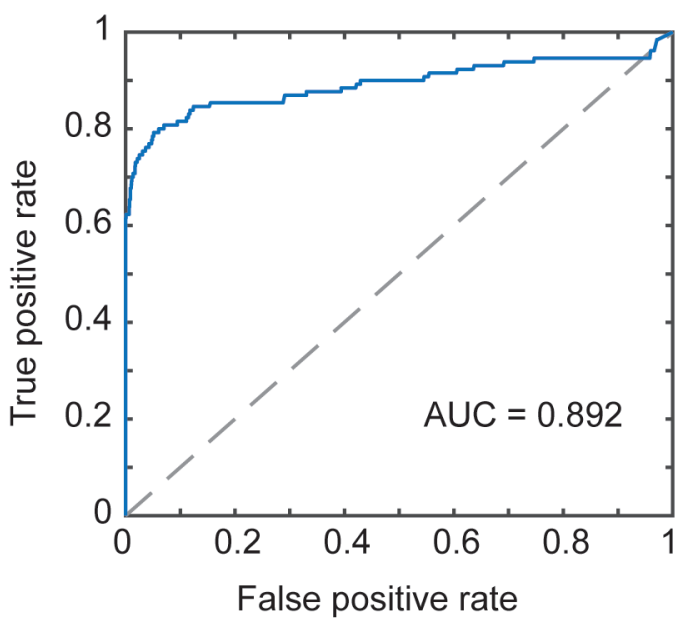

b
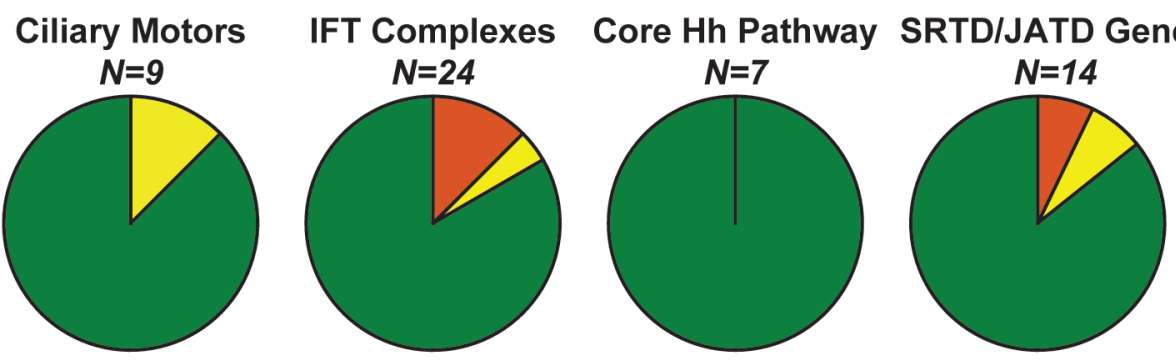

$10 \%$ FDR hit

$\square 20 \%$ FDR hit

$\square$ non-hit

N.D.
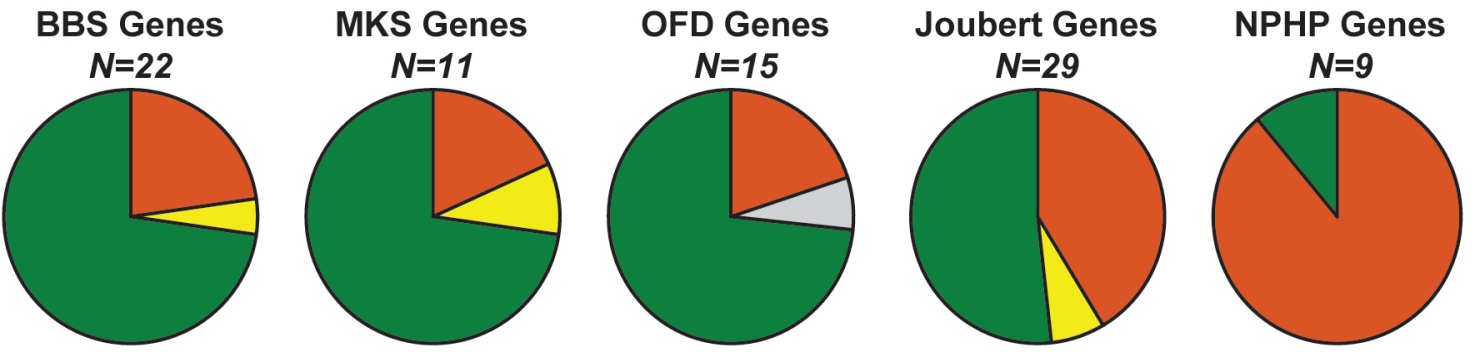

C

\section{Core Cilia/ciliopathy Genes}

$N=88$

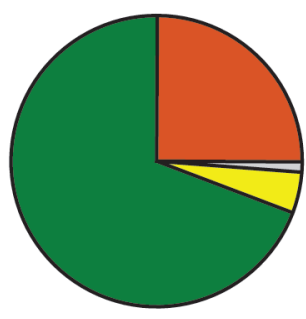

This study

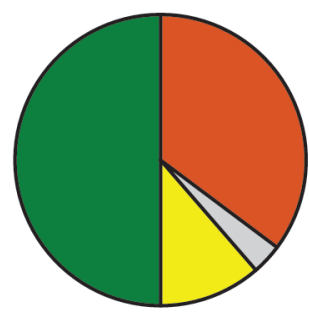

Roosing et al. (ciliogenesis)

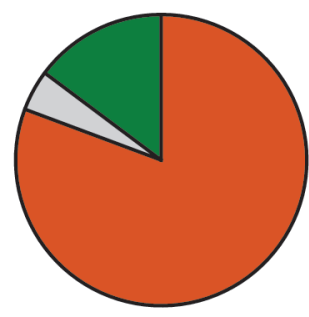

Wheway et al. (ciliogenesis)

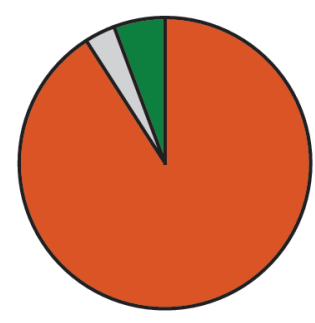

Jacob et al. (Hh signaling) 


\section{Figure 4}

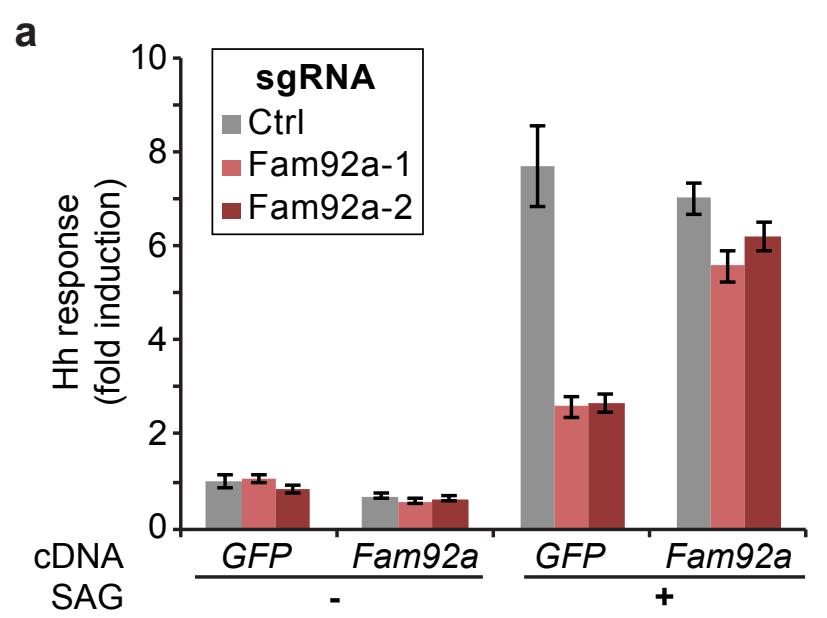

e

b

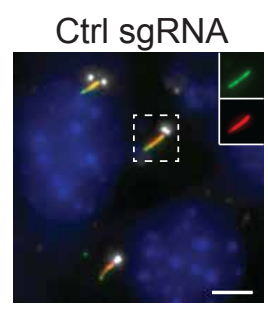

Fam92a sgRNA-2

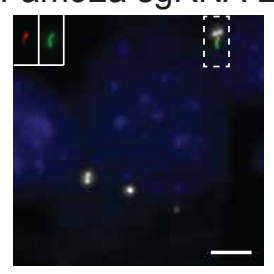

ARL13B

Yotulbulin

Ac-tubulin

DNA

C

$\begin{array}{cccc}\text { Protein } & \begin{array}{c}\text { NSAF } \\ \text { (spectra } \\ \text { per 100aa) }\end{array} & \begin{array}{c}\text { coverage } \\ \text { (\%) }\end{array} & \begin{array}{c}\text { Screen rank } \\ \text { (percentile) }\end{array} \\ \text { FAM92A-LAP } & 38.9 & 50 & 99.8 \\ \text { CBY1 } & 11.0 & 50 & 99.7 \\ \text { DZIP1L } & 2.1 & 10 & 90.2\end{array}$

d
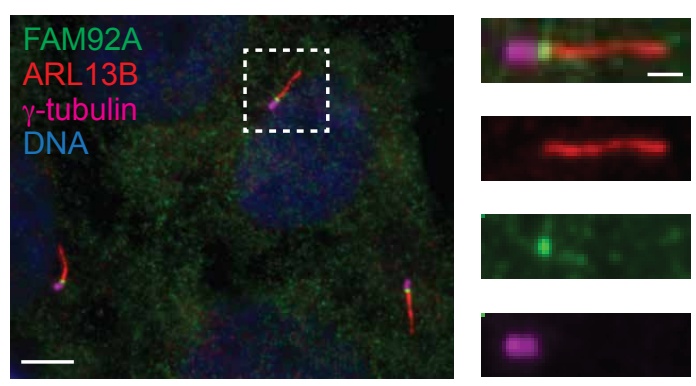

$\begin{array}{cccc}\text { Protein } & \begin{array}{c}\text { NSAF } \\ \text { (spectra } \\ \text { per 100aa) }\end{array} & \begin{array}{c}\text { coverage } \\ \text { (\%) }\end{array} & \begin{array}{c}\text { Screen rank } \\ \text { (percentile) }\end{array} \\ \text { TTC23-LAP } & 64.3 & 60 & 99.0 \\ \text { IQCE } & 28.6 & 55 & 97.1 \\ \text { EFCAB7 } & 18.5 & 50 & 97.6\end{array}$

f
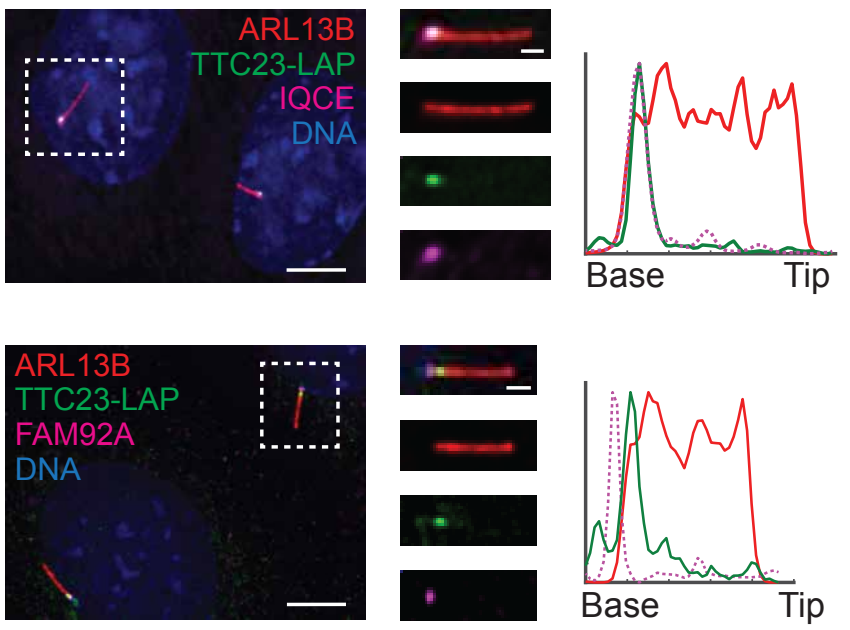

g

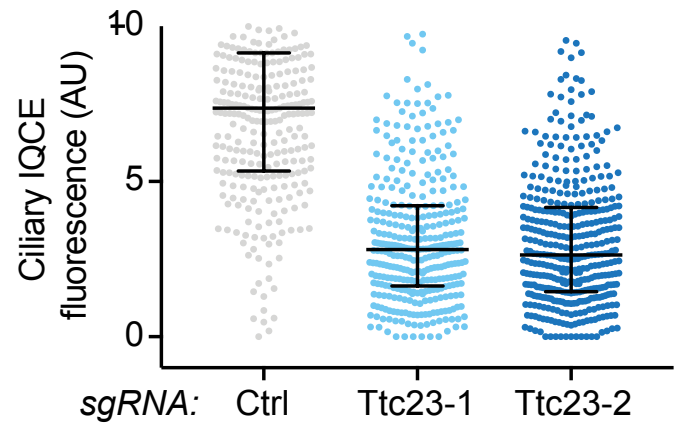

h

siCtrl1
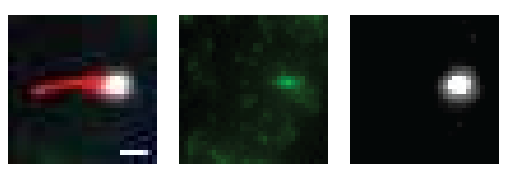

TTC23 (GFP) IQCE ARL13B DNA

silqce
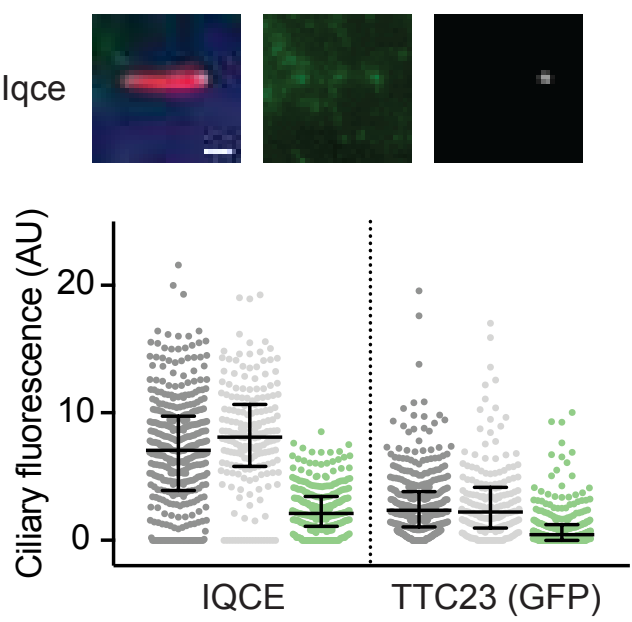

siCtrl1

siCtrl2

silqce 

aCC-BY-NC 4.0 International license.

\section{Figure 5}

a
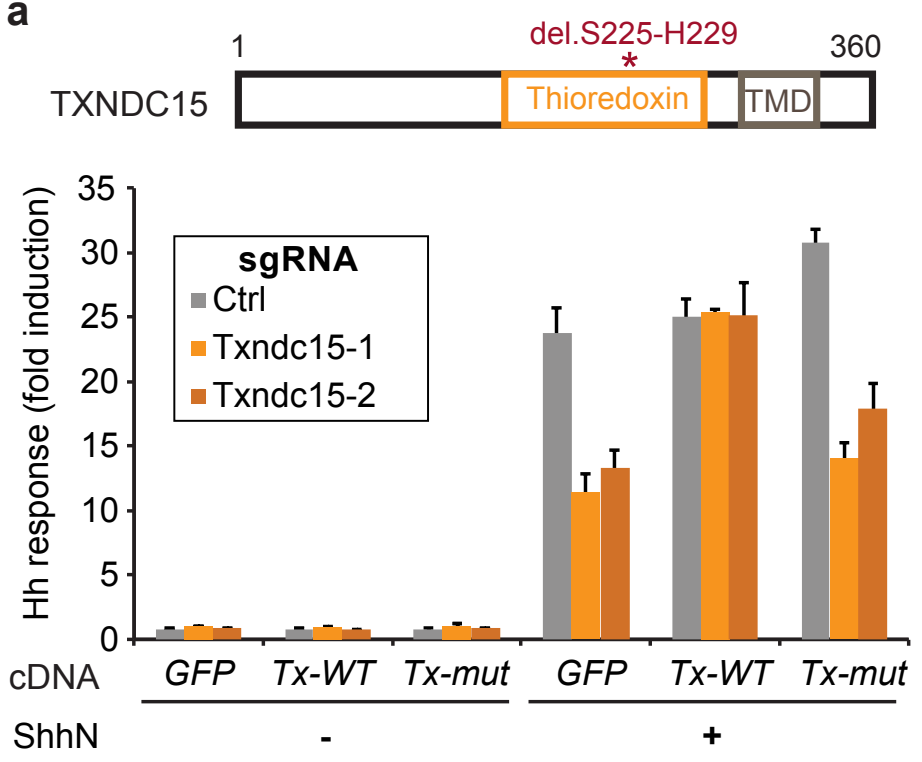

b
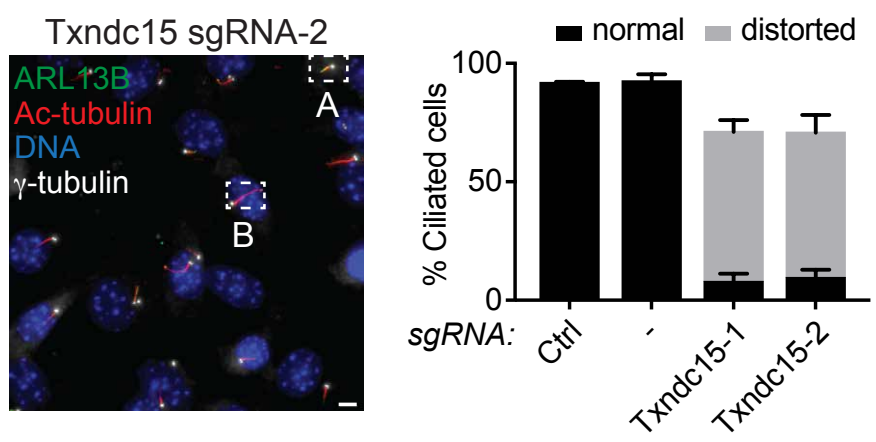

A (normal):

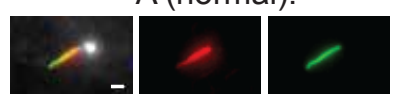

B (distorted):

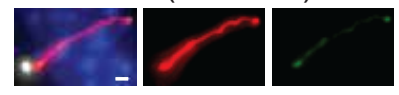

d

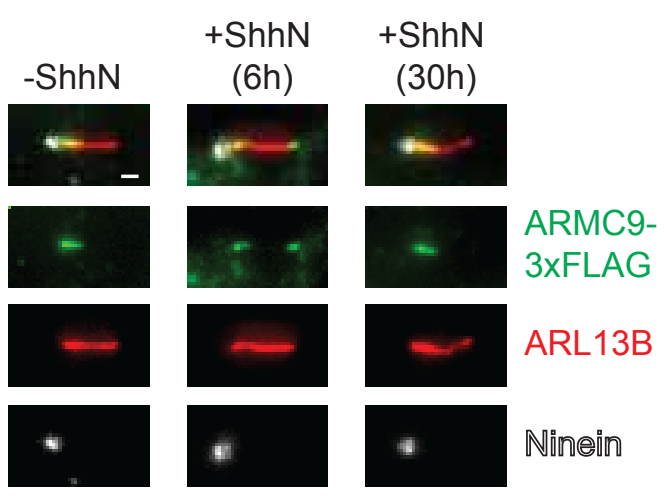

e

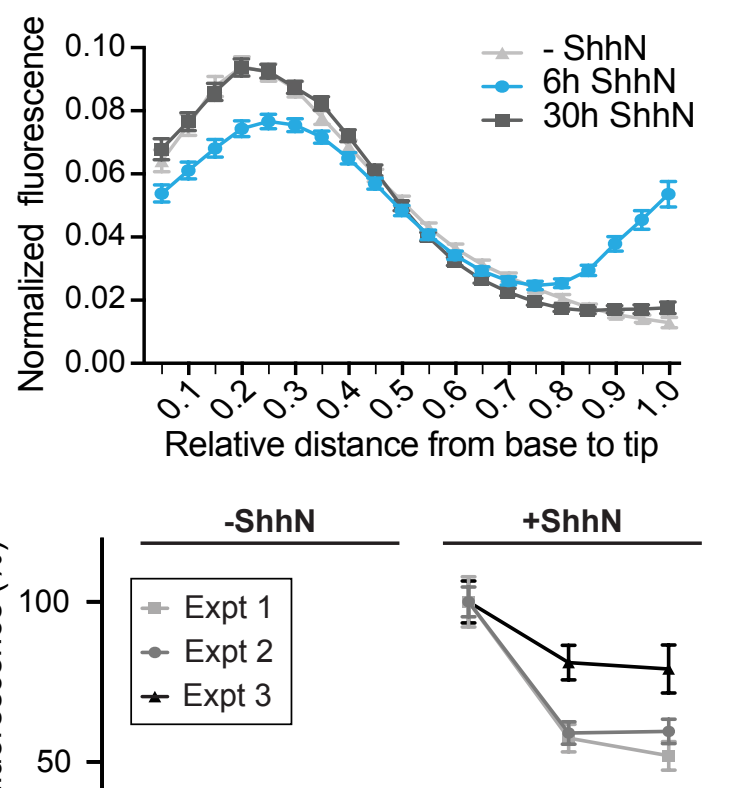

f

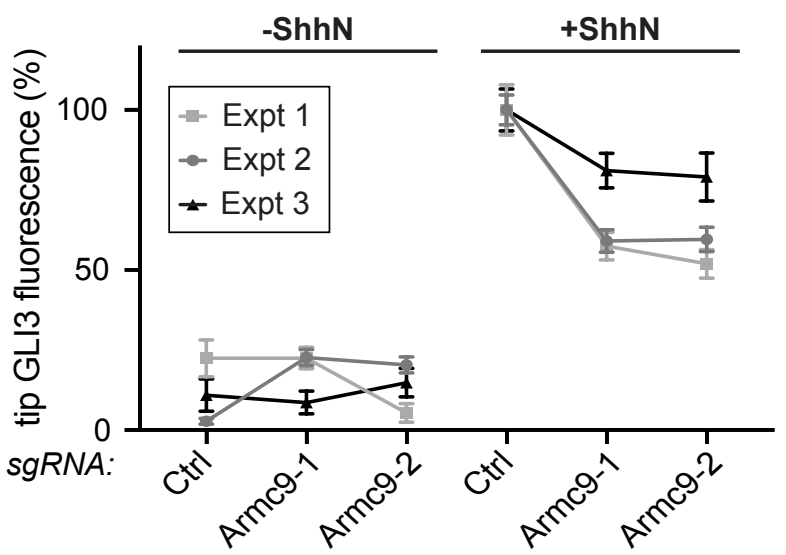

e
C
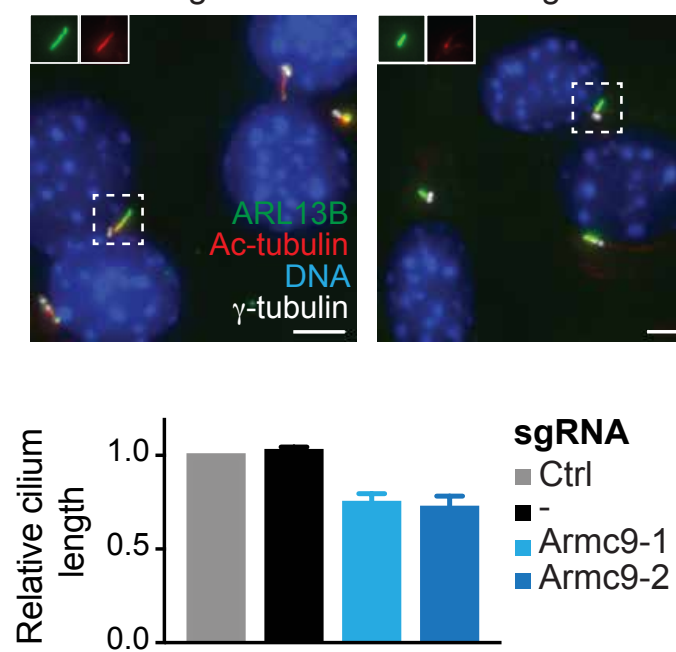

g

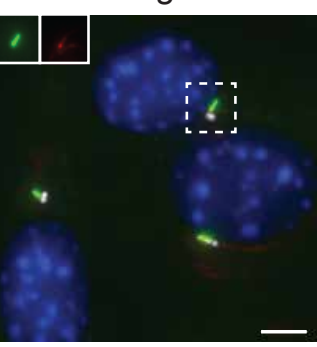

\begin{tabular}{r} 
Renal disease \\
\hline Developmental delay \\
Obesity
\end{tabular}

Digital anomalies

Retinopathy

Skeletal dysplasia

Heart defects

Craniofacial defects

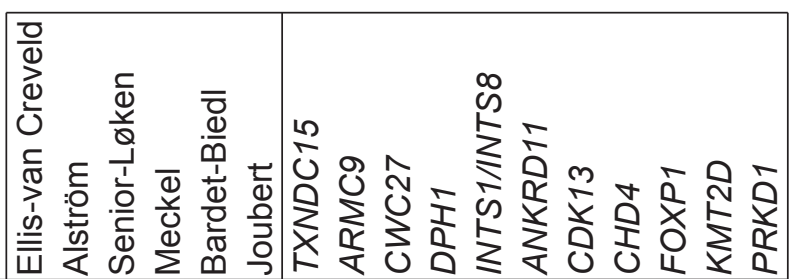


bioRxiv preprint doi: https://doi.org/10.1101/156059; this version posted June 27, 2017. The copyright holder for this preprint (which was not certified by peer review) is the author/funder, who has granted bioRxiv a license to display the preprint in perpetuity. It is made available under

Figure 6

a

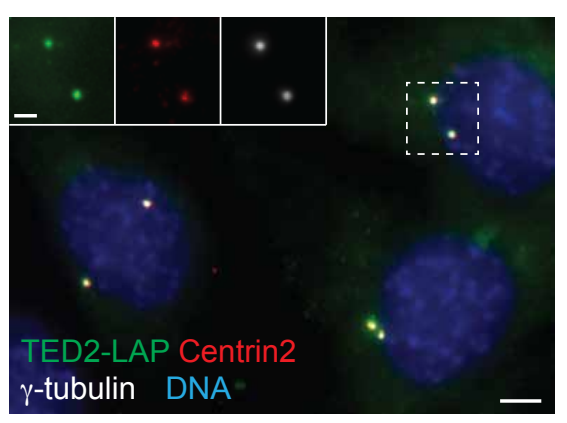

b

$\begin{array}{cccc}\text { PSAF } & \begin{array}{c}\text { NSAF } \\ \text { (spectra } \\ \text { per 100aa) }\end{array} & \begin{array}{c}\text { coverage } \\ \text { (\%) }\end{array} & \begin{array}{c}\text { Screen rank } \\ \text { (percentile) }\end{array} \\ \text { TED2-LAP } & 46.8 & 76 & 99.5 \\ \text { TED1 } & 47.1 & 73 & 99.8 \\ \text { TUBE1 } & 32.6 & 61 & 98.7 \\ \text { TUBD1 } & 30.3 & 60 & 99.4 \\ \text { TUBB5 } & 14.4 & 54 & \text { lethal } \\ \text { TUBA1B } & 12.9 & 55 & \text { lethal } \\ \text { CENPJ } & 4.0 & 35 & 85.2\end{array}$

C

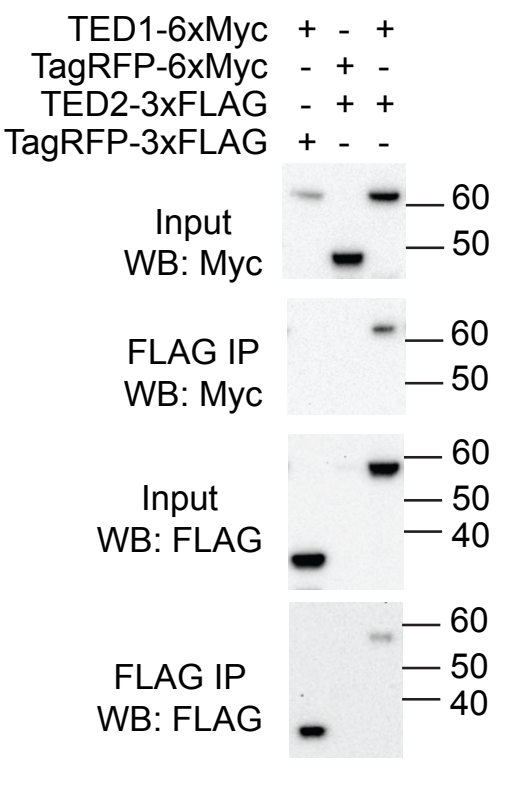



aCC-BY-NC 4.0 International license.

\section{Figure 7}

a
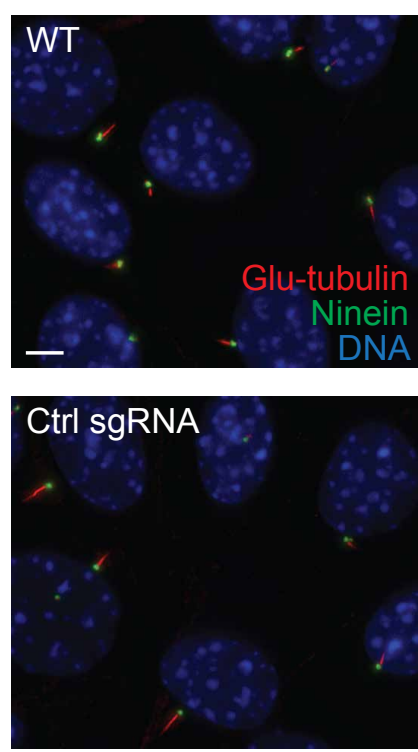
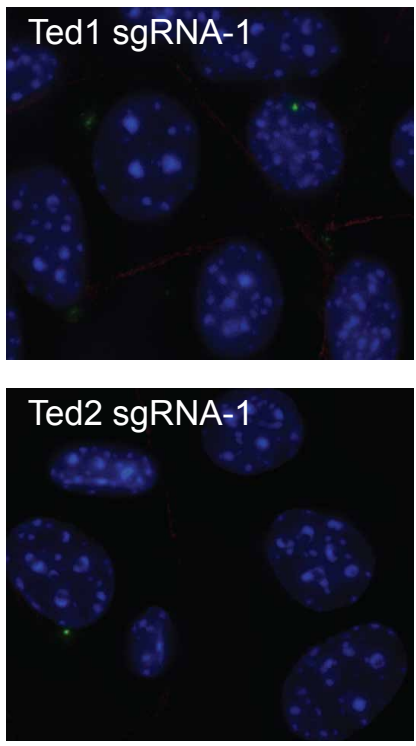
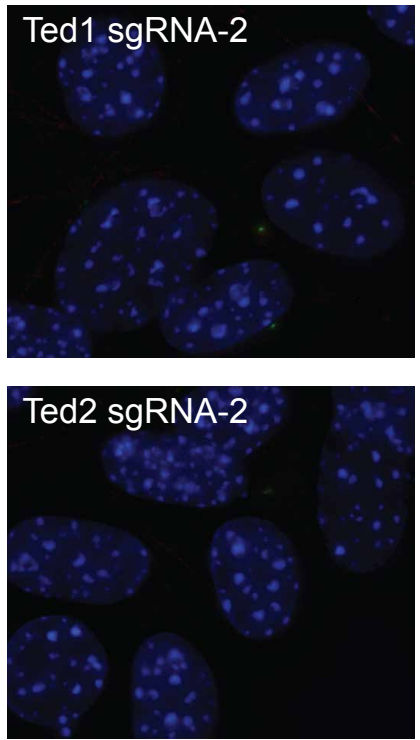

b

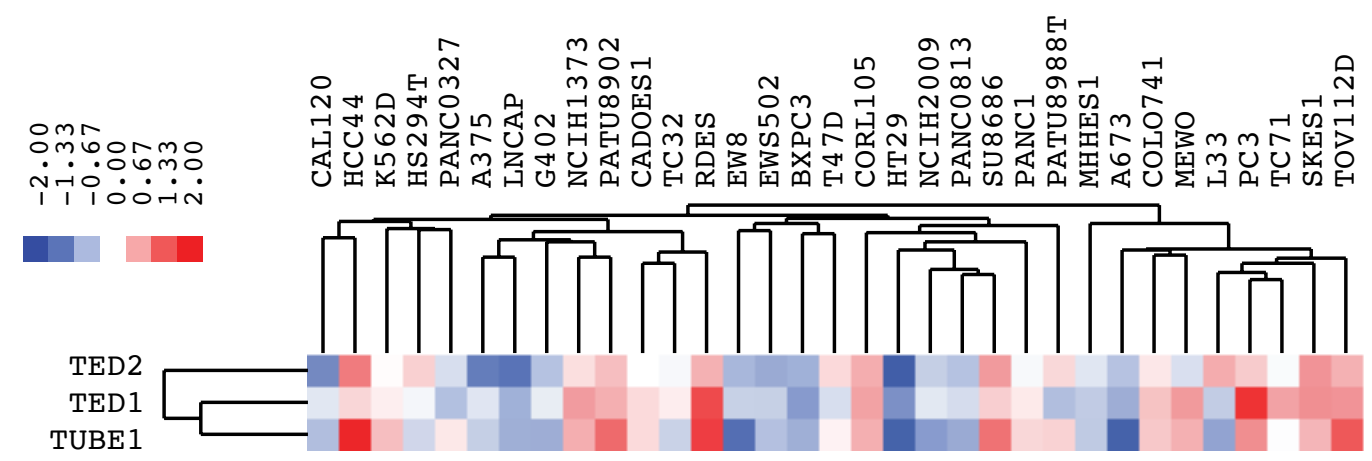

C
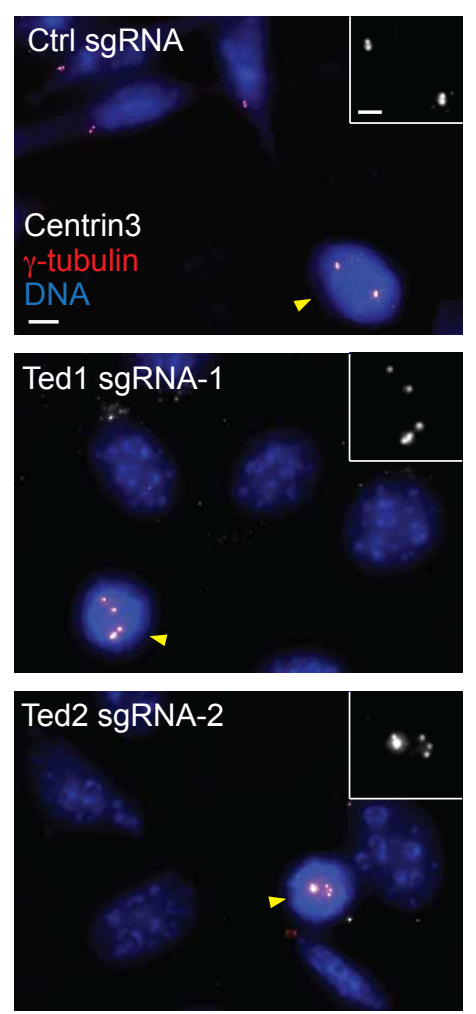

d

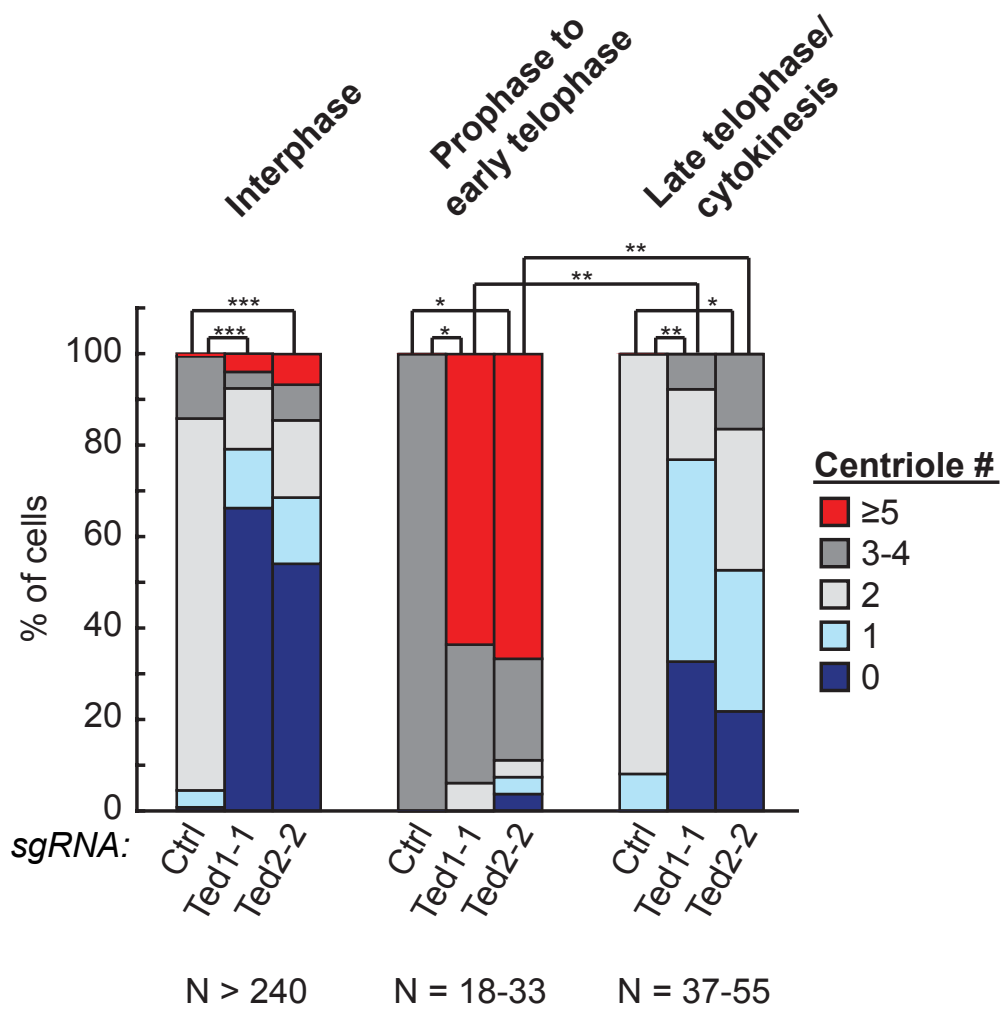

